\title{
Multiple-Modal-Coupling Modeling and Stability Analysis of Cold Rolling Mill Vibration
}

\author{
Lingqiang Zeng, Yong Zang, and Zhiying Gao \\ School of Mechanical Engineering, University of Science and Technology Beijing, Beijing 100083, China \\ Correspondence should be addressed to Zhiying Gao; gaozhiying@me.ustb.edu.cn
}

Received 3 February 2016; Revised 13 May 2016; Accepted 24 May 2016

Academic Editor: Athanasios Chasalevris

Copyright (c) 2016 Lingqiang Zeng et al. This is an open access article distributed under the Creative Commons Attribution License, which permits unrestricted use, distribution, and reproduction in any medium, provided the original work is properly cited.

\begin{abstract}
An effective dynamic model is the basis for studying rolling mill vibration. Through analyzing characteristics of different types of vibration, a coupling vibration structure model is established, in which vertical vibration, horizontal vibration, and torsional vibration can be well indicated. In addition, based on the Bland-Ford-Hill rolling force model, a dynamic rolling process model is formulated. On this basis, the rolling mill vertical-torsional-horizontal coupled dynamic model is constructed by coupling the rolling process model and the mill structure model. According to this mathematical model, the critical rolling speed is determined and the accuracy of calculated results is verified by experimental data. Then, the interactions between different subsystems are demonstrated by dynamic responses in both time and frequency domains. Finally, the influences of process parameters and structure parameters on system stability are analyzed. And a series of experiments are conducted to verify the correctness of these analysis conclusions. The results show that the vertical-torsional-horizontal coupled model can reasonably characterize the coupling relationship between the mill structure and the rolling process. These studies are helpful for formulating a reasonable technological procedure of the rolling process and determining a feasible dynamic modification strategy of the structure as well.
\end{abstract}

\section{Introduction}

Strips are the most widely used rolled products. With the increase of steel productions and the further optimization of the steel product mix, the industrial demand for strips has been growing, and the requirement for the product quality has become increasingly strict. However, the improvement of the production efficiency and the product quality is seriously restricted due to the presence of mill vibration.

Generally, three kinds of typical vibration have been observed in rolling by scholars. They are identified as vertical vibration, torsional vibration, and horizontal vibration. And the vertical vibration is classified into two types: third-octavemode chatter $(120 \sim 250 \mathrm{~Hz})$ and fifth-octave-mode chatter $(500 \sim 700 \mathrm{~Hz})$.

Third-octave-mode chatter is considered to be the most harmful vibration phenomenon that occurred in high-speed cold tandem mills. It is characterized by a sudden occurrence, which tends to accumulate a lot of energy in a few seconds to make the vibration amplitude increase rapidly. A four-high mill structure model proposed by Yarita et al. [1] is the earliest model used to study the third-octave-mode chatter. Tamiya et al. [2] studied the third-octave-mode chatter on a cold rolling mill and reported that this type of vibration is a self-excited vibration due to the phase difference between the vertical movement of rolls and the strip tension at entry. On this basis, Yun et al. [3], Hu et al. [4], and Zhao and Ehmann [5], the scholars of Northwestern University, conducted a series of systematic studies on vibration of rolling mills. Yun et al. [3] reported that negative damping, mode coupling, and regeneration were the three possible causes of third-octavemode chatter. Hu et al. [4] further developed the dynamic rolling process model based on the study of Yun and studied the correlation between different rolling parameters and the stability of the system. Zhao and Ehmann [5] researched the third-octave-mode chatter based on a multistand model and found that the dynamic characteristics of this system are more complex due to the regenerative effect. Meehan [6] studied the third-octave-mode chatter on a 4-high rolling mill and pointed that the roll stack damping and the tension stress have the most effect on the critical speed. 
Compared with third-octave-mode chatter, fifth-octavemode chatter causes less damage to rolls and strips. But it affects the product appearance. Roberts [7] explained the mechanism of fifth-octave-mode chatter and its negative impacts in detail and put forward a series of measures to suppress fifth-octave-mode chatter. $\mathrm{Hu}$ and Ehmann [8] studied the critical condition in which the system would become unstable owing to fifth-octave-mode chatter.

Torsional vibration may cause fatigue effect of transmission system components or even instantaneous damage. Gallenstein [9] studied the possible causes of the torsional vibration on a test cold mill and discussed the quantitative relationship between rolling process parameters and the rolling torque. Krot [10] created a drive transmission structure model of the rolling mill and presented a diagnostic method for torsional vibration impact strength and equipment wear.

In addition, Paton and Critchley [11] found that rolls can vibrate not only in vertical direction, but also in horizontal direction. Shen and $\mathrm{Li}$ [12] pointed that the horizontal vibration would result in minor fluctuations of the roll gap, which would lead to the occurrence of abnormal excitation forces and break the equilibrium state of the system. Dwivedy et al. [13] also reported that horizontal vibration may do serious harm to rolling mills and quality of strips. It may cause light and dark marks on the work roll surface or even cause accidents such as the strip breakage and the piling-up of steel.

In recent years, specialists and scholars have done a lot of researches on rolling mill vibration and have gained abundant achievements. But these studies mostly focused on the vibration of a single subsystem like torsional subsystem or vertical subsystem. Only a few scholars studied the coupling vibration of rolling mills. Swiatoniowski [14] proposed a typical vertical-torsional coupling structure model with a two-high rolling mill as an object. Yan et al. [15] studied the vertical-torsional coupling vibration using FEM and found that the torsional vibration may strengthen the vibration of vertical subsystem. Hou et al. [16] proposed a verticalhorizontal coupling model based on the interactions between the dynamic rolling force and the mill structure and found that the system parameters have significant effect on the rolling mill coupling vibration. Yun et al. [17] proposed a model which allows rolls to vibrate along two orthogonal directions to study the vertical-horizontal coupling vibration and suggested that mode coupling might also induce vibration. Since the vibration of a high-speed rolling mill always appears as the coupling of multiple vibration types, it becomes a hot topic to study multiple-modal-coupling vibration.

As reported by Tlusty et al. [18], mill vibration is caused by the interaction between the rolling mill structure and the rolling process. Based on this interaction, [19] has constructed an overall coupled model of the rolling mill, but the calculation of the neutral point position is not sufficiently precise, and the stability analysis is not systematic. Therefore, a further research needs to be performed. In this paper, a vertical-torsional-horizontal coupling vibration model of the rolling mill is modeled according to the relationship shown in Figure 1. The structure model, in which vertical

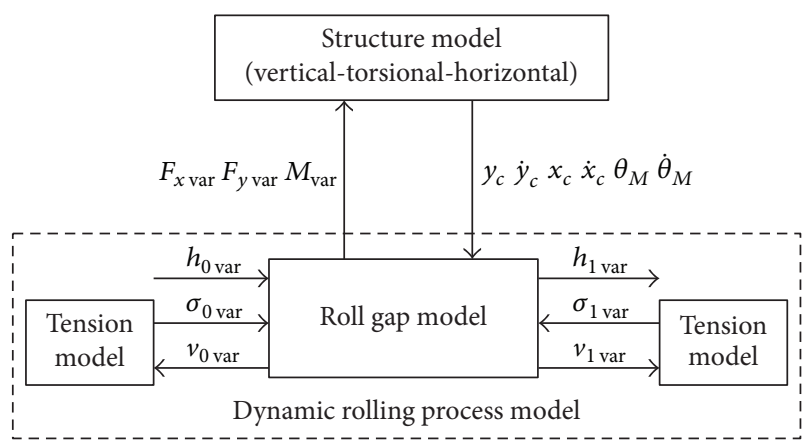

FIGURE 1: Coupling relationship of structural model and rolling process model.

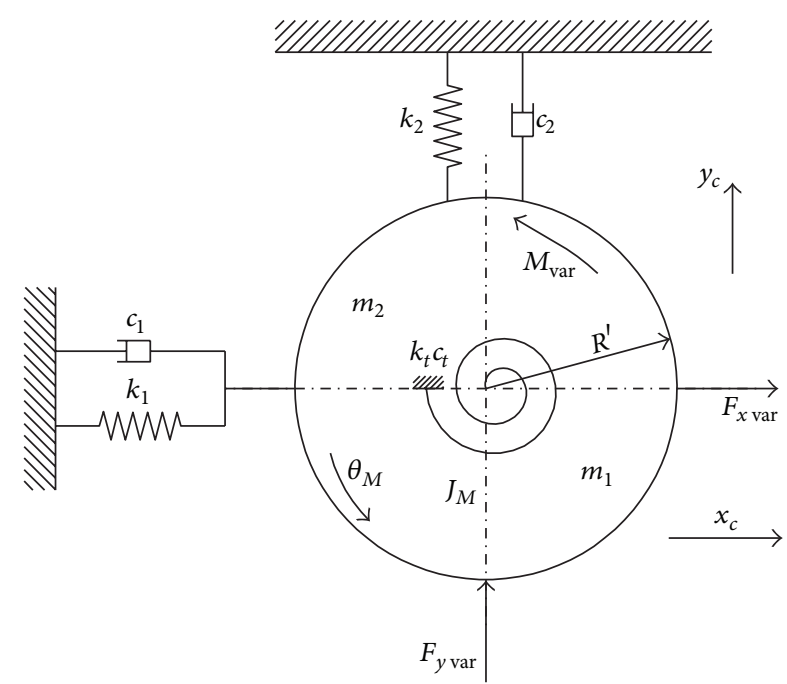

FIGURE 2: Simplified vertical-torsional-horizontal coupling structural model.

vibration, horizontal, vibration and torsional vibration can be well indicated, is established under the assumption that the vibration is symmetrical with respect to the center plane of the strip. The dynamic rolling process model, which can be used to calculate the strip velocities, tensions, and forces acting on the rolls, is constructed based on the Bland-FordHill rolling force model. According to this mathematic model, the critical rolling speed of the system is calculated, the interaction between the different subsystems is discussed, and the influence of process parameters and structure parameters on the system stability is analyzed.

\section{Structure Model}

The three most typical strip rolling mills are two-high mill, four-high mill, and six-high mill. Each of them can be simplified to a mass-spring system. In order to facilitate the analysis, the mill structure is assumed to be symmetrical with respect to the center plane of the strip, and the vertical subsystem, horizontal subsystem, and torsional subsystem are all simplified as ones with single degree of freedom. The coupling structure model is illustrated in Figure 2. 


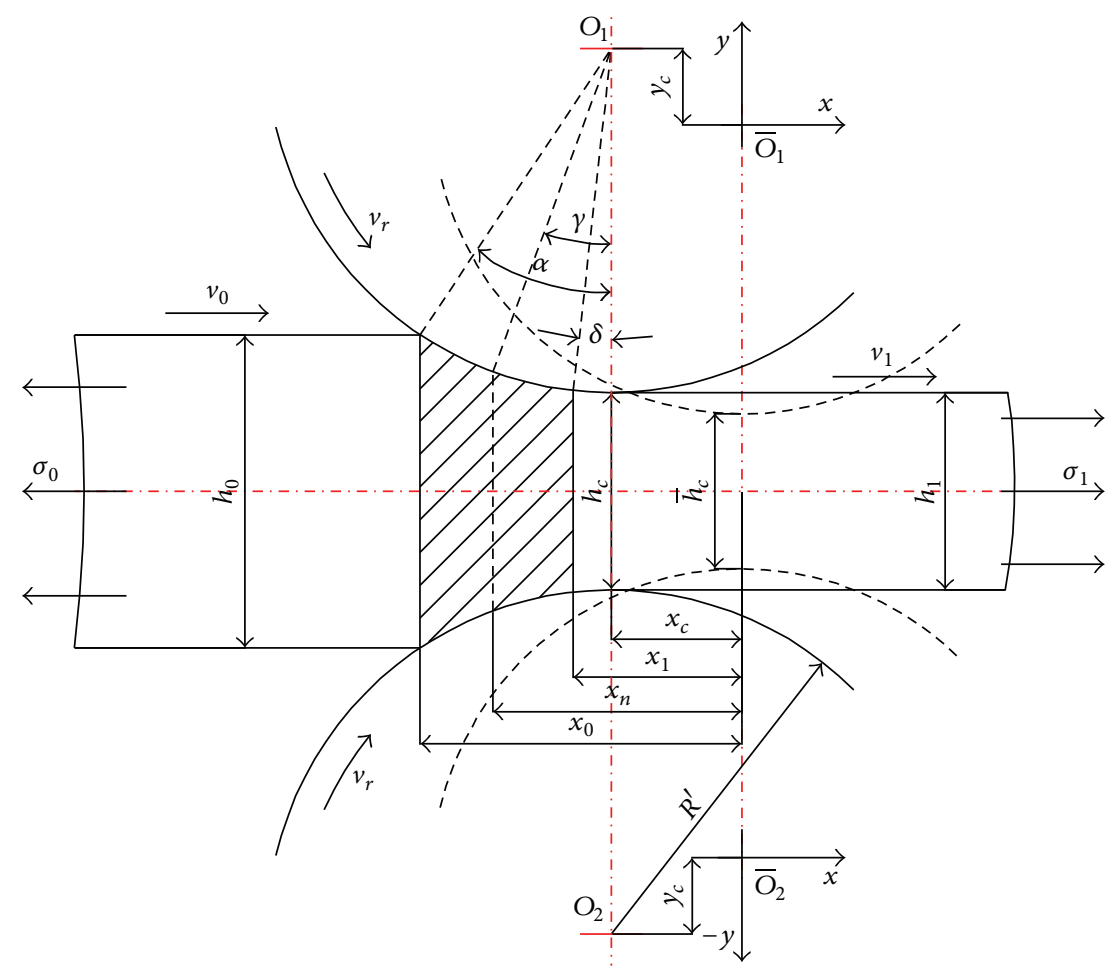

FIgURE 3: Roll bite geometry during vibration.

In this structural model, only the top work roll is allowed to vibrate in horizontal direction, and the impact of the top backup roll on horizontal vibration is reflected in the support stiffness. For vertical subsystem, the top work roll, the top backup roll, and the upper mill housing are treated as a whole, as their vibration in vertical direction is in phase. The model is effective when studying third-octave-mode chatter.

As this structural model is symmetrical, it is only valid to study the symmetrical vibration of the rolling mill. For asymmetric vibration, an asymmetrical model is needed. In that case, the rolling process model and corresponding equations will be more complicated absolutely.

As shown in Figure 2, $m_{1}$ is the equivalent mass of the top work roll. $m_{2}$ is the lumped equivalent mass of the top work roll, top backup roll, and upper mill housing, which is simplified to the center of the top work roll. $J_{M}$ is the equivalent rotational inertia of the upper rolls, including the top work roll and the top backup roll. $k_{1}, c_{1}$ are the horizontal equivalent stiffness and horizontal equivalent damping due to the support from unilateral pillars of the rolling mill and the support from the top backup roll to the top work roll because of the offset between them. $k_{2}, c_{2}$ are the vertical equivalent stiffness and vertical equivalent damping due to the support from the frame and the upper beam of the rolling mill. $k_{t}, c_{t}$ are the torsional equivalent stiffness and torsional equivalent damping of the top main drive system. $R^{\prime}$ is the radius of the deformed top work roll. $x_{c}$ is the displacement of horizontal vibration. $y_{c}$ is the displacement of vertical vibration. $\theta_{M}$ is the angular displacement of torsional vibration.

Thus the differential equations can be written as follows:

$$
\begin{gathered}
m_{1} \ddot{x}_{c}+c_{1} \dot{x}_{c}+k_{1} x_{c}=F_{x \mathrm{var}}, \\
m_{2} \ddot{y}_{c}+c_{2} \dot{y}_{c}+k_{2} y_{c}=F_{y \mathrm{var}}, \\
J_{M} \ddot{\theta}_{M}+c_{t} \dot{\theta}_{M}+k_{t} \theta_{M}=M_{\mathrm{var}},
\end{gathered}
$$

where $F_{x \text { var }}$ and $F_{y \text { var }}$ are the fluctuations of forces acting on the rolls in $x$ and $y$ directions and $M_{\mathrm{var}}$ is the fluctuation of the rolling torque.

\section{Dynamic Model of Rolling Process}

\subsection{Theoretical Model of Dynamic Rolling Process}

3.1.1. Roll Bite Geometry. The geometry of the roll bite is schematically presented in Figure 3 , where $\bar{O}_{1}$ and $\bar{O}_{2}$ are the original geometrical centers of the top and bottom rolls (before vibrations begin). $\mathrm{O}_{1}$ and $\mathrm{O}_{2}$ are the ever-changing centers of the rolls. $x_{c}$ is the distance from the centerline $\mathrm{O}_{1} \mathrm{O}_{2}$ to the centerline $\bar{O}_{1} \bar{O}_{2} . h_{0}$ and $h_{1}$ are the thicknesses of the strip at entry and exit. $h_{c}$ is the roll gap spacing measured along the centerline $\mathrm{O}_{1} \mathrm{O}_{2} . v_{0}$ and $v_{1}$ are the velocities of the strip at entry and exit. $v_{r}$ is the roll peripheral velocity. $x_{0}, x_{n}$, and $x_{1}$ are the entry position, neutral point position, and exit position measured from the centerline $\bar{O}_{1} \bar{O}_{2} . \sigma_{0}$ and $\sigma_{1}$ are the tensions of the strip at entry and exit. $\alpha, \gamma$, and $\delta$ are the inlet angle, the neutral angle, and the exit angle. As shown 
in Figure 3, the rolls of the top and the bottom can vibrate in $x, y$, and rotation directions symmetrically. In addition, it is assumed that the width of the strip always keeps as $B$ during rolling.

As shown in Figure 3, the dynamic roll gap spacing can be written as follows:

$$
h_{c}=\bar{h}_{c}+2 y_{c}
$$

where the parameters with superscript like " $i$ " represent the value of parameter " $i$ " under steady state.

In the rolling deformation zone, the roll surface was usually treated as a parabolic curve, so the strip thickness at any arbitrary position is written as follows:

$$
h(x)=h_{c}+\frac{\left(x-x_{c}\right)^{2}}{R^{\prime}} .
$$

During the vibration of rolls, the volume of the roll bite changes simultaneously. Considering the control volume of the material flow within the roll bite, $\mathrm{Hu}$ et al. [4] modified the metal flow equation and expressed it as follows:

$$
v h=v_{0} h_{0}-2 \dot{y}_{c}\left(x-x_{0}\right)-\dot{x}_{c}\left(h_{0}-h\right),
$$

where $v$ and $h$ are the strip velocity and thickness of any cross section in the roll bite. The second and third terms on the right hand side of (4) represent the material flow fluctuations of any cross section caused by the vibration of rolls in $y$ and $x$ directions.

For the cold rolling, the rolling pressure is particularly high, so the work roll will be elastically flattened obviously. Here, the Hitchcock equation is used to calculate the effective flattened radius of the work roll:

$$
R^{\prime}=R\left(1+\frac{2 c}{\Delta h} \frac{P}{B}\right)
$$

where $\Delta h$ is the reduction, $\Delta h=h_{0}-h_{1}$ and $c=8\left(1-u_{2}^{2}\right) / \pi E_{2}$. $E_{2}$ and $u_{2}$ are Young's modulus and Poisson's ratio of the roll.

As the function relationship between the flattened work roll radius and the rolling force, it is very difficult to decouple them. In order to simplify the analysis, the original radius is replaced by the effective radius, which is calculated iteratively during computations of the static rolling process. In addition, it is assumed that the effective radius of the work roll keeps unchanged along the contact arc between the work roll and the strip. These measures and assumptions not only can help to improve the accuracy of calculations but also can obtain a solvable analytical model.

3.1.2. Strip Entry Position and Exit Position. The entry position of the strip can be derived from (3) as follows:

$$
x_{0}=x_{c}-\sqrt{R^{\prime}\left(h_{0}-h_{c}\right)} .
$$

From the geometric relationship shown in Figure 3, the inlet angle is expressed as follows:

$$
\alpha=\arcsin \frac{x_{c}-x_{0}}{R^{\prime}}=\arcsin \sqrt{\frac{h_{0}-h_{c}}{R^{\prime}}} .
$$

Substituting $h(x)$ from (3) into (4), the horizontal velocity of the strip at any vertical cross-section plane within the roll bite can be expressed as follows:

$$
v(x)=\frac{v_{0} h_{0}-2 \dot{y}_{c}\left(x-x_{0}\right)-\dot{x}_{c}\left(h_{0}-h(x)\right)}{h_{c}+\left(x-x_{c}\right)^{2} / R^{\prime}} .
$$

The strain rate of the strip at any vertical cross-section plane within the roll bite can be derived from (3):

$$
\begin{aligned}
\frac{d h}{d t} & =\frac{\partial h}{\partial t}+\frac{\partial h}{\partial x} \frac{\partial x}{\partial t}+\frac{\partial h}{\partial x_{c}} \frac{\partial x_{c}}{\partial t} \\
& =\frac{\partial h_{c}}{\partial t}+\frac{2\left(x-x_{c}\right)}{R^{\prime}}\left(v(x)-\dot{x}_{c}\right) .
\end{aligned}
$$

The exit position of the strip can be determined by examining the condition of zero strain rates (Zhao and Ehmann [5]), so

$$
\frac{\partial h_{c}}{\partial t}+\frac{2\left(x_{1}-x_{c}\right)}{R^{\prime}}\left(v_{1}-\dot{x}_{c}\right)=0
$$

Substituting (2) and (8) into (10) and rewriting it,

$$
\begin{aligned}
\dot{y}_{c}\left(x_{1}-x_{c}\right)^{2} & \\
& -\left[v_{0} h_{0}-2 \dot{y}_{c}\left(x_{c}-x_{0}\right)-\dot{x}_{c} h_{0}\right]\left(x_{1}-x_{c}\right) \\
& -R^{\prime} h_{c} \dot{y}_{c}=0 .
\end{aligned}
$$

Neglecting the quadratic term $\left(x_{1}-x_{c}\right)^{2}$, then the exit position of the strip can be represented as follows:

$$
x_{1}=x_{c}-\frac{R^{\prime} h_{c} \dot{y}_{c}}{v_{0} h_{0}-2 \dot{y}_{c}\left(x_{c}-x_{0}\right)-\dot{x}_{c} h_{0}} .
$$

It can be seen from the above equation that the exit plane no longer coincides with the centerline of the rolls as the occurrence of vibration, so the exit angle can be expressed as follows:

$$
\delta=\arcsin \frac{x_{c}-x_{1}}{R^{\prime}}
$$

3.1.3. Friction Model. The change of the friction along the contact arc is very complicated. It neither obeys the dry friction law nor obeys the adhesive friction theory [20]. Since the resultant force on the rolling interface is the focus in the study of mill vibration, the selected friction model should be simple, accurate, and solvable. 


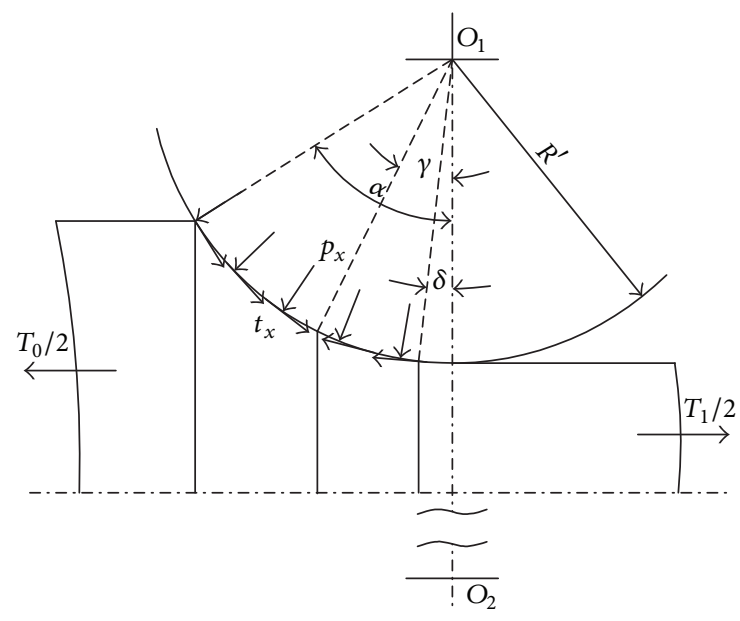

FIGURE 4: Equilibrium of force system in the roll bite.

In cold rolling, vibration often happens on the last few stands, and friction coefficients for these stands are usually very small. In this case, the friction force is far lower than the shear strength of the strip material, which means the friction state is sliding friction and there is no sticking point in the deformation zone. Therefore, Coulomb friction model can be used in the study of vibration in cold rolling. In order to simplify the analysis, we assume that the friction coefficients along the contact arc keep unchanged and are represented as $\mu_{m}$.

3.1.4. Neutral Point Position. In general, there is a point on the contact arc of the rolling deformation zone, named neutral point, where the peripheral velocity of the work roll and the velocity of the strip are equal. The neutral point is one of the most important variables in the rolling process. Along the contact arc, the friction changes direction on different sides of this point.

As shown in Figure 4, the neutral angle can be determined by the equilibrium condition of the force system in the rolling deformation zone:

$$
\begin{array}{r}
-\int_{\delta}^{\alpha} p_{x} \sin \alpha_{x} R^{\prime} d \alpha_{x}+\int_{\gamma}^{\alpha} \mu_{m} p_{x} \cos \alpha_{x} R^{\prime} d \alpha_{x} \\
-\int_{\delta}^{\gamma} \mu_{m} p_{x} \cos \alpha_{x} R^{\prime} d \alpha_{x}+\frac{T_{1}-T_{0}}{2 B}=0,
\end{array}
$$

where $T_{1}$ and $T_{2}$ are tensile forces of the strip at entry and exit, $T_{1}=\sigma_{1} h_{1} B$, and $T_{0}=\sigma_{0} h_{0} B . \alpha_{x}$ is the central angle corresponding to any arbitrary vertical cross section. $p_{x}$ is the radial unit pressure, and we assume that $p_{x}$ along the contact arc is uniform distribution. So it can be approximated that $p_{x}$ is equal to the mean unit pressure $p_{m}, p_{x} \approx p_{m}=P /(B l)$, and $l$ is the horizontal projection length of the contact arc, $l=x_{1}-x_{0}$.

From (14), the neutral angle can be formulated as follows:

$$
\sin \gamma=\frac{\sin \alpha+\sin \delta}{2}+\frac{\cos \alpha-\cos \delta}{2 \mu_{m}}+\frac{\left(T_{1}-T_{0}\right) l}{4 \mu_{m} P R^{\prime}} .
$$

From the geometric relationship shown in Figure 3, the position of the neutral point is expressed as follows:

$$
\begin{aligned}
x_{n}= & x_{c}-R^{\prime} \sin \gamma \\
= & x_{c}-\frac{R^{\prime}}{2}(\sin \alpha+\sin \delta)-\frac{R^{\prime}}{2 \mu_{m}}(\cos \alpha-\cos \delta) \\
& -\frac{\left(T_{1}-T_{0}\right) l}{4 \mu_{m} P} .
\end{aligned}
$$

3.1.5. Strip Velocities at Entry and Exit. Once the position of the neutral point is obtained, the strip velocity at any arbitrary vertical cross section of the roll bite can be also determined. As the strip velocity at the neutral point equals the roll peripheral velocity, substituting $x_{n}$ from (16) into (8), the strip velocity at the neutral point can be expressed as follows:

$$
v_{r}=\frac{v_{0} h_{0}-2 \dot{y}_{c}\left(x_{n}-x_{0}\right)-\dot{x}_{c}\left(h_{0}-h_{n}\right)}{h_{c}+\left(x_{n}-x_{c}\right)^{2} / R^{\prime}} .
$$
follows:

Thus, the strip velocities at entry and exit are written as

$$
\begin{aligned}
v_{0} & =\frac{1}{h_{0}}\left[\left(v_{r}-\dot{x}_{c}\right)\left(h_{c}+\frac{\left(x_{n}-x_{c}\right)^{2}}{R^{\prime}}\right)+2 \dot{y}_{c}\left(x_{n}-x_{0}\right)\right. \\
& \left.+h_{0} \dot{x}_{c}\right], \\
v_{1} & =\frac{v_{0} h_{0}-2 \dot{y}_{c}\left(x_{1}-x_{0}\right)-\dot{x}_{c}\left(h_{0}-h_{c}-\left(x_{1}-x_{c}\right)^{2} / R^{\prime}\right)}{h_{c}+\left(x_{1}-x_{c}\right)^{2} / R^{\prime}} .
\end{aligned}
$$

3.1.6. Calculation of Rolling Force. The rolling force is one of the most important energetic parameters of the rolling mill. Specialists and scholars have done a lot of research on the rolling force and also achieved a variety of rolling force models for different mills or working conditions. Reference [21] indicated that most of the cold rolling mills can meet the conditions of Bland-Ford-Hill rolling force model. The form of Hill simplified formula is as follows:

$$
P=\left(K-\sigma_{m}\right) B Q_{p} l,
$$

where $\sigma_{m}$ is the mean tension, usually taking $\sigma_{m}=0.7 \sigma_{0}+$ $0.3 \sigma_{1} \cdot Q_{p}$ is the stress state factor. $K$ is the mean deformation resistance.

The stress state factor of Hill simplified formula is expressed as follows:

$$
Q_{p}=1.08+1.79 \varepsilon \mu \sqrt{\frac{R^{\prime}}{h_{0}}}-1.02 \varepsilon,
$$


where $\varepsilon$ is the reduction ratio, expressed as $\varepsilon=\left(h_{0}-h_{1}\right) / h_{0}$. $\mu$ is the friction coefficient along the contact arc, taking $\mu=\mu_{m}$.

In the rolling mill, the strip tensions at entry and exit have a significant effect on the stability of the system. And the variations of strip tensions have a direct relationship with variations of strip velocities. As the vibration characteristic of a single stand mill is the focus of this paper, supposing both the variation of the exit velocity in the upstream stand and the variation of the entry velocity in the downstream stand are zero, tension variations at entry and exit can be expressed according to Hooke's law as follows:

$$
\begin{gathered}
\sigma_{0 \mathrm{var}}=\frac{E_{1}}{L_{0}} \Delta L_{0}=\frac{E_{1}}{L_{0}} \int_{0}^{t}\left(v_{0}-\bar{v}_{0}\right) d t, \\
\sigma_{1 \mathrm{var}}=\frac{E_{1}}{L_{1}} \Delta L_{1}=-\frac{E_{1}}{L_{1}} \int_{0}^{t}\left(v_{1}-\bar{v}_{1}\right) d t,
\end{gathered}
$$

where $E_{1}$ is Young's modulus of the strip. $L_{0}$ and $L_{1}$ are the distances from this stand to the upstream stand and the downstream stand.

Hence, the dynamic tensions of the strip at entry and exit can be written as follows:

$$
\begin{aligned}
& \sigma_{0}=\bar{\sigma}_{0}+\sigma_{0 \mathrm{var}} \\
& \sigma_{1}=\bar{\sigma}_{1}+\sigma_{1 \mathrm{var}} .
\end{aligned}
$$

In addition, the strain hardening effect must be considered when calculating the deformation resistance of the cold-rolled strip. Because of the strain hardening effect, the deformation resistance of each rolling pass is not only related to the deformation extent of this rolling pass but also associated with the total deformation extent of rolling passes before. Furthermore, the deformation extent is also changing along the contact arc. As a consequence, the mean total deformation extent is used to calculate the mean deformation resistance (Zou [22]). Consider

$$
\varepsilon_{\sum_{m}}=b_{0} \varepsilon_{0}+b_{1} \varepsilon_{1}
$$

where $\varepsilon_{0}$ is the total deformation extent at the entry of this mill stand, $\varepsilon_{0}=\left(H-h_{0}\right) / H$. $\varepsilon_{1}$ is the total deformation extent at the exit of this mill stand, $\varepsilon_{1}=\left(H-h_{1}\right) / H$. $H$ is the initial strip thickness entering the first mill stand in a tandem mill configuration. $b_{0}$ and $b_{1}$ are coefficients, usually taking $b_{0}=$ 0.4 and $b_{1}=0.6$.

For cold rolling, the following equation can be used to calculate the mean deformation resistance:

$$
K=A \cdot \varepsilon_{\sum_{m}}^{n}
$$

where $A$ and $n$ are the coefficients associated with the carbon content of the strip.
3.1.7. Forces Acting on the Rolls and the Rolling Torque. As shown in Figure 4, forces acting on the rolls in $x$ and $y$ directions can be expressed as follows:

$$
\begin{aligned}
F_{x} & =B \int_{\delta}^{\alpha} p_{x} \sin \alpha_{x} R^{\prime} d \alpha_{x} \\
& +B\left(\int_{\delta}^{\gamma} \mu_{m} p_{x} \cos \alpha_{x} R^{\prime} d \alpha_{x}\right. \\
& \left.-\int_{\gamma}^{\alpha} \mu_{m} p_{x} \cos \alpha_{x} R^{\prime} d \alpha_{x}\right)=\frac{T_{1}-T_{0}}{2} \\
& =\frac{\sigma_{1} h_{1} B-\sigma_{0} h_{0} B}{2}, \\
F_{y} & =B \int_{\delta}^{\alpha} p_{x} \cos \alpha_{x} R^{\prime} d \alpha_{x} \\
& -B\left(\int_{\delta}^{\gamma} \mu_{m} p_{x} \sin \alpha_{x} R^{\prime} d \alpha_{x}\right. \\
& \left.-\int_{\gamma}^{\alpha} \mu_{m} p_{x} \sin \alpha_{x} R^{\prime} d \alpha_{x}\right)=B R^{\prime} p_{x}(\sin \alpha-\sin \delta) \\
& -B R^{\prime} \mu_{m} p_{x}(\cos \alpha+\cos \delta-2 \cos \gamma)=P \\
& -B R^{\prime} \mu_{m} p_{x}(\cos \alpha+\cos \delta-2 \cos \gamma) .
\end{aligned}
$$

As the angles $\alpha, \gamma$, and $\delta$ are very small, the second term on the right hand side of (27) can be negligible; that is,

$$
F_{y}=P=\left(K-\sigma_{m}\right) B Q_{p} l .
$$

The rolling torque is expressed as follows:

$$
\begin{aligned}
M & =B\left(\int_{\delta}^{\gamma} \mu_{m} p_{x} R R^{\prime} d \alpha_{x}-\int_{\gamma}^{\alpha} \mu_{m} p_{x} R R^{\prime} d \alpha_{x}\right) \\
& =\mu_{m} p_{x} B R R^{\prime}(2 \gamma-\alpha-\delta)=-\frac{(\alpha+\delta-2 \gamma)}{\alpha-\delta} \mu_{m} P R .
\end{aligned}
$$

3.2. Linearization of the Dynamic Rolling Process Model. Although the theoretical model of the dynamic rolling process has been established in the previous section, it is not easy to study the rolling vibration analytically because of its nonlinear nature. Therefore, a linearization process is done by using a first-order Taylor series approximation for all the factors of interest, and then the resulting variations are used to formulate a linearized dynamic rolling process model.

In this model, the variables like $\sigma_{0}, \sigma_{1}, h_{0}, y_{c}, \dot{y}_{c}, x_{c}, \dot{x}_{c}$, and $\dot{\theta}_{M}$ are defined as inputs; therefore, the variation of any linearized model output can then be expressed as follows:

$$
\begin{aligned}
Q_{i \mathrm{var}}= & \left(\frac{\partial Q_{i}}{\partial \sigma_{0}}\right)_{s} \sigma_{0 \mathrm{var}}+\left(\frac{\partial Q_{i}}{\partial \sigma_{1}}\right)_{s} \sigma_{1 \mathrm{var}} \\
& +\left(\frac{\partial Q_{i}}{\partial h_{0}}\right)_{s} h_{0 \mathrm{var}}+\left(\frac{\partial Q_{i}}{\partial y_{c}}\right)_{s} y_{c}+\left(\frac{\partial Q_{i}}{\partial \dot{y}_{c}}\right)_{s} \dot{y}_{c} \\
& +\left(\frac{\partial Q_{i}}{\partial \dot{\theta}_{M}}\right)_{s} \dot{\theta}_{M}+\left(\frac{\partial Q_{i}}{\partial x_{c}}\right)_{s} x_{c}+\left(\frac{\partial Q_{i}}{\partial \dot{x}_{c}}\right)_{s} \dot{x}_{c},
\end{aligned}
$$


where the subscript " $s$ " designates that the corresponding derivative is evaluated at steady-state conditions.

3.2.1. Variations of Strip Entry and Exit Positions. From (6), the variation of strip entry position can be expressed as follows:

$$
\begin{aligned}
x_{0 \mathrm{var}} & =\left(\frac{\partial x_{0}}{\partial h_{0}}\right)_{s} h_{0 \mathrm{var}}+\left(\frac{\partial x_{0}}{\partial h_{c}}\right)_{s} h_{c \mathrm{var}}+\left(\frac{\partial x_{0}}{\partial x_{c}}\right)_{s} x_{c} \\
& =\left(\frac{\partial x_{0}}{\partial h_{0}}\right)_{s} h_{0 \mathrm{var}}+2\left(\frac{\partial x_{0}}{\partial h_{c}}\right)_{s} y_{c}+\left(\frac{\partial x_{0}}{\partial x_{c}}\right)_{s} x_{c} .
\end{aligned}
$$

Substituting $v_{0}$ from (18) into (12), the strip exit position is written as follows:

$$
\begin{aligned}
& x_{1} \\
& =x_{c} \\
& \quad-\frac{R^{\prime} h_{c} \dot{y}_{c}}{\left(v_{r}-\dot{x}_{c}\right)\left(h_{c}+\left(x_{n}-x_{c}\right)^{2} / R^{\prime}\right)+2 \dot{y}_{c}\left(x_{n}-x_{c}\right)} .
\end{aligned}
$$

Therefore, the variation of strip exit position is expressed as follows:

$$
\begin{aligned}
x_{1 \mathrm{var}}= & 2\left(\frac{\partial x_{1}}{\partial h_{c}}\right)_{s} y_{c}+\left(\frac{\partial x_{1}}{\partial \dot{y}_{c}}\right)_{s} \dot{y}_{c}+\left(\frac{\partial x_{1}}{\partial v_{r}}\right)_{s} R^{\prime} \dot{\theta}_{M} \\
& +\left(\frac{\partial x_{1}}{\partial x_{n}}\right)_{s} x_{n \mathrm{var}}+\left(\frac{\partial x_{1}}{\partial x_{c}}\right)_{s} x_{c}+\left(\frac{\delta x_{1}}{\delta \dot{x}_{c}}\right)_{s} \dot{x}_{c} .
\end{aligned}
$$

3.2.2. Variation of Neutral Point Position. Substituting $P$ from (20) into (16), the variation of neutral point position can then be calculated as follows:

$$
\begin{aligned}
x_{n \mathrm{var}}= & \left(\frac{\partial x_{n}}{\partial \sigma_{0}}\right)_{s} \sigma_{0 \mathrm{var}}+\left(\frac{\partial x_{n}}{\partial \sigma_{1}}\right)_{s} \sigma_{1 \mathrm{var}} \\
& +\left(\frac{\partial x_{n}}{\partial h_{0}}\right)_{s} h_{0 \mathrm{var}}+\left(\frac{\partial x_{n}}{\partial h_{1}}\right)_{s} h_{1 \mathrm{var}} \\
& +\left(\frac{\partial x_{n}}{\partial x_{c}}\right)_{s} x_{c}+\left(\frac{\partial x_{n}}{\partial \alpha}\right)_{s} \alpha_{\mathrm{var}}+\left(\frac{\partial x_{n}}{\partial \delta}\right)_{s} \delta_{\mathrm{var}} \\
& +\left(\frac{\partial x_{n}}{\partial K}\right)_{s} K_{\mathrm{var}}+\left(\frac{\partial x_{n}}{\partial \sigma_{m}}\right)_{s} \sigma_{m \mathrm{var}} \\
& +\left(\frac{\partial x_{n}}{\partial Q_{p}}\right)_{s} Q_{p \mathrm{var}} .
\end{aligned}
$$

It can be seen from (34) that some of the items are implicit expressions of independent variables, and they must be converted to explicit expressions. Substituting $x_{1}$ from (32) into (3), the strip thickness at exit is written as follows:

$$
\begin{aligned}
h_{1} & =h_{c} \\
& +\frac{R^{\prime} h_{c}^{2} \dot{y}_{c}^{2}}{\left[\left(v_{r}-\dot{x}_{c}\right)\left(h_{c}+\left(x_{n}-x_{c}\right)^{2} / R^{\prime}\right)+2 \dot{y}_{c}\left(x_{n}-x_{c}\right)\right]^{2}} .
\end{aligned}
$$

Then the variation of strip thickness at exit is expressed as follows:

$$
\begin{aligned}
h_{1 \mathrm{var}}= & 2\left(\frac{\partial h_{1}}{\partial h_{c}}\right)_{s} y_{c}+\left(\frac{\partial h_{1}}{\partial \dot{y}_{c}}\right)_{s} \dot{y}_{c}+\left(\frac{\partial h_{1}}{\partial v_{r}}\right)_{s} R^{\prime} \dot{\theta}_{M} \\
& +\left(\frac{\partial h_{1}}{\partial x_{n}}\right)_{s} x_{n \mathrm{var}}+\left(\frac{\partial h_{1}}{\partial x_{c}}\right)_{s} x_{c}+\left(\frac{\delta h_{1}}{\delta \dot{x}_{c}}\right)_{s} \dot{x}_{c} .
\end{aligned}
$$

As the inlet angle and the exit angle are small in cold rolling, approximately taking $\alpha \approx \sin \alpha=\sqrt{\left(h_{0}-h_{c}\right) / R^{\prime}}$ and $\delta \approx \sin \delta=\left(x_{c}-x_{1}\right) / R^{\prime}$, hence their first-order Taylor series approximations are expressed as follows:

$$
\begin{aligned}
& \alpha_{\mathrm{var}}=\left(\frac{\partial \alpha}{\partial h_{0}}\right)_{s} h_{0 \mathrm{var}}+2\left(\frac{\partial \alpha}{\partial h_{c}}\right)_{s} y_{c}, \\
& \delta_{\mathrm{var}}=\left(\frac{\partial \delta}{\partial x_{c}}\right)_{s} x_{c}+\left(\frac{\partial \delta}{\partial x_{1}}\right)_{s} x_{1 \mathrm{var}} .
\end{aligned}
$$

And the variations of the mean deformation resistance, the mean tension, and the stress state factor are formulated as follows:

$$
\begin{aligned}
K_{\mathrm{var}} & =\left(\frac{\partial K}{\partial h_{0}}\right)_{s} h_{0 \mathrm{var}}+\left(\frac{\partial K}{\partial h_{1}}\right)_{s} h_{1 \mathrm{var}}, \\
\sigma_{m \mathrm{var}} & =\left(\frac{\partial \sigma_{m}}{\partial \sigma_{0}}\right)_{s} \sigma_{0 \mathrm{var}}+\left(\frac{\partial \sigma_{m}}{\partial \sigma_{1}}\right)_{s} \sigma_{1 \mathrm{var}} \\
& =0.7 \sigma_{0 \mathrm{var}}+0.3 \sigma_{1 \mathrm{var}}, \\
Q_{p \mathrm{var}} & =\left(\frac{\partial Q_{p}}{\partial h_{0}}\right)_{s} h_{0 \mathrm{var}}+\left(\frac{\partial Q_{p}}{\partial h_{1}}\right)_{s} h_{1 \mathrm{var}} .
\end{aligned}
$$

Substituting (36) -(41) into (34), the resulting equation for the variation of neutral point position becomes

$$
\begin{aligned}
x_{n \mathrm{var}}= & a_{x_{n}, \sigma_{0}} \sigma_{0 \mathrm{var}}+a_{x_{n}, \sigma_{1}} \sigma_{1 \mathrm{var}}+a_{x_{n}, h_{0}} h_{0 \mathrm{var}} \\
& +a_{x_{n}, y_{c}} y_{c}+a_{x_{n}, y_{c}} \dot{y}_{c}+a_{x_{n}, x_{c}} x_{c} .
\end{aligned}
$$


3.2.3. Variations of Strip Velocities at Entry and Exit. From (18) and (19), the variations of strip velocities at entry and exit are expressed as follows:

$$
\begin{aligned}
v_{0 \mathrm{var}}= & \left(\frac{\partial v_{0}}{\partial h_{0}}\right)_{s} h_{0 \mathrm{var}}+2\left(\frac{\partial v_{0}}{\partial h_{c}}\right)_{s} y_{c}+\left(\frac{\partial v_{0}}{\partial \dot{y}_{c}}\right)_{s} \dot{y}_{c} \\
& +\left(\frac{\partial v_{0}}{\partial x_{c}}\right)_{s} x_{c}+\left(\frac{\partial v_{0}}{\partial \dot{x}_{c}}\right)_{s} \dot{x}_{c} \\
& +\left(\frac{\partial v_{0}}{\partial v_{r}}\right)_{s} R^{\prime} \dot{\theta}_{M}+\left(\frac{\partial v_{0}}{\partial x_{n}}\right)_{s} x_{n \mathrm{var}} \\
& +\left(\frac{\partial v_{0}}{\partial x_{0}}\right)_{s} x_{0 \mathrm{var}}, \\
v_{1 \mathrm{var}}= & \left(\frac{\partial v_{1}}{\partial h_{0}}\right)_{s} h_{0 \mathrm{var}}+2\left(\frac{\partial v_{1}}{\partial h_{c}}\right)_{s} y_{c}+\left(\frac{\partial v_{1}}{\partial \dot{y}_{c}}\right)_{s} \dot{y}_{c} \\
& +\left(\frac{\partial v_{1}}{\partial x_{c}}\right)_{s} x_{c}+\left(\frac{\partial v_{1}}{\partial \dot{x}_{c}}\right)_{s} \dot{x}_{c}+\left(\frac{\partial v_{1}}{\partial v_{0}}\right)_{s} v_{0 \mathrm{var}} \\
& +\left(\frac{\partial v_{1}}{\partial x_{1}}\right)_{s} x_{1 \mathrm{var}}+\left(\frac{\partial v_{1}}{\partial x_{0}}\right)_{s} x_{0 \mathrm{var}}
\end{aligned}
$$

Therefore, the explicit expressions of (43) and (44) can be written as follows:

$$
\begin{aligned}
v_{0 \mathrm{var}}= & a_{v_{0}, \sigma_{0}} \sigma_{0 \mathrm{var}}+a_{v_{0}, \sigma_{1}} \sigma_{1 \mathrm{var}}+a_{v_{0}, h_{0}} h_{0 \mathrm{var}}+a_{v_{0}, y_{c}} y_{c} \\
& +a_{v_{0}, \dot{y}_{c}} \dot{y}_{c}+a_{v_{0}, x_{c}} x_{c}+a_{v_{0}, \dot{x}_{c}} \dot{x}_{c}+a_{v_{0}, \dot{\theta}_{M}} \dot{\theta}_{M}, \\
v_{1 \mathrm{var}}= & a_{v_{1}, \sigma_{0}} \sigma_{0 \mathrm{var}}+a_{v_{1}, \sigma_{1}} \sigma_{1 \mathrm{var}}+a_{v_{1}, h_{0}} h_{0 \mathrm{var}}+a_{v_{1}, y_{c}} y_{c} \\
& +a_{v_{1}, \dot{y}_{c}} \dot{y}_{c}+a_{v_{1}, x_{c}} x_{c}+a_{v_{1}, \dot{x}_{c}} \dot{x}_{c}+a_{v_{1}, \dot{\theta}_{M}} \dot{\theta}_{M} .
\end{aligned}
$$

\subsubsection{Variations of Forces Acting on the Rolls and the Rolling} Torque. Applying the first-order Taylor series approximation to (26), the variation of the force acting on the rolls in $x$ direction is expressed as follows:

$$
\begin{aligned}
F_{x \mathrm{var}}= & \left(\frac{\partial F_{x}}{\partial \sigma_{0}}\right)_{s} \sigma_{0 \mathrm{var}}+\left(\frac{\partial F_{x}}{\partial \sigma_{1}}\right)_{s} \sigma_{1 \mathrm{var}} \\
& +\left(\frac{\partial F_{x}}{\partial h_{0}}\right)_{s} h_{0 \mathrm{var}}+\left(\frac{\partial F_{x}}{\partial h_{1}}\right)_{s} h_{1 \mathrm{var}} \\
= & a_{F_{x}, \sigma_{0}} \sigma_{0 \mathrm{var}}+a_{F_{x}, \sigma_{1}} \sigma_{1 \mathrm{var}}+a_{F_{x}, h_{0}} h_{0 \mathrm{var}} \\
& +a_{F_{x}, y_{c}} y_{c} .
\end{aligned}
$$

Likewise, as the variation of horizontal projection length of the contact arc is $l_{\mathrm{var}}=x_{1 \mathrm{var}}-x_{0 \mathrm{var}}$ and considering (33) and (39)-(42), the variation of the force acting on the rolls in $y$ direction can be derived from (28):

$$
\begin{aligned}
F_{y \text { var }}= & \left(\frac{\partial F_{y}}{\partial K}\right)_{s} K_{\mathrm{var}}+\left(\frac{\partial F_{y}}{\partial \sigma_{m}}\right)_{s} \sigma_{m \mathrm{var}} \\
& +\left(\frac{\partial F_{y}}{\partial Q_{p}}\right)_{s} Q_{p \mathrm{var}}+\left(\frac{\partial F_{y}}{\partial l}\right)_{s} l_{\mathrm{var}} \\
= & a_{F_{y}, \sigma_{0}} \sigma_{0 \mathrm{var}}+a_{F_{y}, \sigma_{1}} \sigma_{1 \mathrm{var}}+a_{F_{y}, h_{0}} h_{0 \mathrm{var}} \\
& +a_{F_{y}, y_{c}} y_{c}+a_{F_{y}, \dot{y}_{c}} \dot{y}_{c}+a_{F_{y}, x_{c}} x_{c} .
\end{aligned}
$$

As the neutral angle is very small, approximately taking $\gamma \approx \sin \gamma=\left(x_{c}-x_{n}\right) / R^{\prime}$, hence its first-order Taylor series approximation is expressed as follows:

$$
\gamma_{\mathrm{var}}=\left(\frac{\partial \gamma}{\partial x_{c}}\right)_{s} x_{c}+\left(\frac{\partial \gamma}{\partial x_{n}}\right)_{s} x_{n \mathrm{var}} .
$$

Therefore, the variation of the rolling torque can then be calculated from (29):

$$
\begin{aligned}
M_{\mathrm{var}}= & \left(\frac{\partial M}{\partial \alpha}\right)_{s} \alpha_{\mathrm{var}}+\left(\frac{\partial M}{\partial \gamma}\right)_{s} \gamma_{\mathrm{var}}+\left(\frac{\partial M}{\partial \delta}\right)_{s} \delta_{\mathrm{var}} \\
& +\left(\frac{\partial M}{\partial K}\right)_{s} K_{\mathrm{var}}+\left(\frac{\partial M}{\partial \sigma_{m}}\right)_{s} \sigma_{m \mathrm{var}} \\
& +\left(\frac{\partial M}{\partial Q_{p}}\right)_{s} Q_{p \mathrm{var}}+\left(\frac{\partial M}{\partial l}\right)_{s} l_{\mathrm{var}} \\
= & a_{M, \sigma_{0}} \sigma_{0 \mathrm{var}}+a_{M, \sigma_{1}} \sigma_{1 \mathrm{var}}+a_{M, h_{0}} h_{0 \mathrm{var}} \\
& +a_{M, y_{c}} y_{c}+a_{M, \dot{y}_{c}} \dot{y}_{c}+a_{M, x_{c}} x_{c} .
\end{aligned}
$$

All the coefficients of the linearized parameters in this section are given in Appendix A.

3.3. Mathematical Model of the Rolling Vibration. As equations in (22) have the integral terms of time, we take them as a derivative with respect to time. Thus they can also be written as follows:

$$
\begin{aligned}
& \frac{d \sigma_{0 \mathrm{var}}}{d t}=\frac{E_{1}}{L_{0}}\left(v_{0}-\bar{v}_{0}\right)=\frac{E_{1}}{L_{0}} v_{0 \mathrm{var}}, \\
& \frac{d \sigma_{1 \mathrm{var}}}{d t}=-\frac{E_{1}}{L_{1}}\left(v_{1}-\bar{v}_{1}\right)=-\frac{E_{1}}{L_{1}} v_{1 \mathrm{var}} .
\end{aligned}
$$


In Section 3.1, $F_{x \mathrm{var}}=F_{x}-\bar{F}_{x} ; F_{y \mathrm{var}}=F_{y}-\bar{F}_{y} ; M_{\mathrm{var}}=$ $M-\bar{M}$; therefore, the mathematical model of rolling vibration based on the theoretical model of the dynamic rolling process is expressed as follows:

$$
\begin{aligned}
m_{1} \ddot{x}_{c}+c_{1} \dot{x}_{c}+k_{1} x_{c} & =F_{x}-\bar{F}_{x}, \\
m_{2} \ddot{y}_{c}+c_{2} \dot{y}_{c}+k_{2} y_{c} & =F_{y}-\bar{F}_{y}, \\
J_{M} \ddot{\theta}_{M}+c_{t} \dot{\theta}_{M}+k_{t} \theta_{M} & =M-\bar{M}, \\
\dot{\sigma}_{0 \mathrm{var}} & =\frac{E_{1}\left(v_{0}-\bar{v}_{0}\right)}{L_{0}},
\end{aligned}
$$

$$
\dot{\sigma}_{1 \mathrm{var}}=\frac{-E_{1}\left(v_{1}-\bar{v}_{1}\right)}{L_{1}} .
$$

And the mathematical model of rolling vibration based on the linearized dynamic rolling process model is expressed as a first-order differential equation in matrix form:

$$
\frac{d X}{d t}=D_{1} X+D_{2} h_{0 \text { var }}
$$

where

$$
\begin{aligned}
X & =\left[\begin{array}{cccccccc}
x_{c} & \dot{x}_{c} & y_{c} & \dot{y}_{c} & \theta_{M} & \dot{\theta}_{M} & \sigma_{0 \mathrm{var}} & \sigma_{1 \mathrm{var}}
\end{array}\right]^{T}, \\
D_{1} & =\left[\begin{array}{cccccccc}
0 & 1 & 0 & 0 & 0 & 0 & 0 & 0 \\
\frac{-k_{1}}{m_{1}} & \frac{-c_{1}}{m_{1}} & \frac{a_{F_{x}, y_{c}}}{m_{1}} & 0 & 0 & 0 & \frac{a_{F_{x}, \sigma_{0}}}{m_{1}} & \frac{a_{F_{x}, \sigma_{1}}}{m_{1}} \\
0 & 0 & 0 & 1 & 0 & 0 & 0 & 0 \\
\frac{a_{F_{y}, x_{c}}}{m_{2}} & 0 & \frac{a_{F_{y}, y_{c}}-k_{2}}{m_{2}} & \frac{a_{F_{y}, \dot{y}_{c}}-c_{2}}{m_{2}} & 0 & 0 & \frac{a_{F_{y}, \sigma_{0}}}{m_{2}} & \frac{a_{F_{y}, \sigma_{1}}}{m_{2}} \\
0 & 0 & 0 & 0 & 0 & 1 & 0 & 0 \\
\frac{a_{M, x_{c}}}{J_{M}} & 0 & \frac{a_{M, y_{c}}}{J_{M}} & \frac{a_{M, \dot{y}_{c}}}{J_{M}} & \frac{-k_{t}}{J_{M}} & \frac{-c_{t}}{J_{M}} & \frac{a_{M, \sigma_{0}}}{J_{M}} & \frac{a_{M, \sigma_{1}}}{J_{M}} \\
\frac{E_{1} a_{v_{0}, x_{c}}}{L_{0}} & \frac{E_{1} a_{v_{0}, \dot{x}_{c}}}{L_{0}} & \frac{E_{1} a_{v_{0}, y_{c}}}{L_{0}} & \frac{E_{1} a_{v_{0}, \dot{y}_{c}}}{L_{0}} & 0 & \frac{E_{1} a_{v_{0}, \dot{\theta}_{M}}}{L_{0}} & \frac{E_{1} a_{v_{0}, \sigma_{0}}}{L_{0}} & \frac{E_{1} a_{v_{0}, \sigma_{1}}}{L_{0}} \\
\frac{-E_{1} a_{v_{1}, x_{c}}}{L_{1}} & \frac{-E_{1} a_{v_{1}, \dot{x}_{c}}}{L_{1}} & \frac{-E_{1} a_{v_{1}, y_{c}}}{L_{1}} & \frac{-E_{1} a_{v_{1}, \dot{y}_{c}}}{L_{1}} & 0 & \frac{-E_{1} a_{v_{1}, \dot{\theta}_{M}}}{L_{1}} & \frac{-E_{1} a_{v_{1}, \sigma_{0}}}{L_{1}} & \frac{-E_{1} a_{v_{1}, \sigma_{1}}}{L_{1}}
\end{array}\right], \\
D_{2} & =\left[\begin{array}{llllll}
0 & \frac{a_{F_{x}, h_{0}}}{m_{1}} & 0 & \frac{a_{F_{y}, h_{0}}}{m_{2}} & 0 & \frac{a_{M, h_{0}}}{J_{M}} \frac{E_{1} a_{v_{0}, h_{0}}}{L_{0}} \frac{-E_{1} a_{v_{1}, h_{0}}}{L_{1}}
\end{array}\right]^{T} \cdot
\end{aligned}
$$

\section{Model Verification}

As the model of rolling mill vibration is formulated by coupling the dynamic rolling process model with the mill structure model, it is necessary to verify the effectiveness of the rolling process model by test data. In addition, in order to study the stability of the mill system, a linearization process for the theoretical rolling process model is done by using a first-order Taylor series approximation; thus it is also necessary to verify the accuracy of the linearized rolling process model.

4.1. Effectiveness of Rolling Process Model. The experimental data are selected from a series of actual rolling parameters in a certain five-stand cold tandem mill. Here the fourth stand is particularly investigated. The setting steady-state values of rolling process parameters are listed in Appendix B, and the invariable parameters are $R=0.28 \mathrm{~m}, \mu_{m}=0.035, n=0.29$, $E_{2}=210 \mathrm{GPa}$, and $u_{2}=0.3$. The rolling force and the rolling torque can be obtained by using equations of Section 3.1. The calculated results are compared with the setting values in
Figure 5, where all the relative errors of the rolling force and the rolling torque are less than $10 \%$. Therefore, the proposed rolling process model can be used to construct the vibration model effectively.

4.2. Accuracy of Linearized Rolling Process Model. Since the linearization process listed in Section 3.2 is a first-order approximation, the variations should not be large, or the results may include an unacceptable amount of error. For this problem, the simulation analysis is carried out on the two mathematical vibration models of Section 3.3. The simulation parameters are listed in Table 1, which are taken from the 4th stand of a 2030 five-stand tandem cold mill.

For the initial condition $y_{c 0}=10^{-6} \mathrm{~m}$, the system dynamic responses based on the theoretical model and the linearized model are shown in Figure 6. It is observed that the theoretical model can be well approximated by the linearized model. The mean absolute errors of following three subgraphs are $6.1 \times 10^{-9} \mathrm{~m}, 2.6 \times 10^{-9} \mathrm{~m}$, and $6.2 \times 10^{-8} \mathrm{~m}$, respectively. And all of them are much smaller than the amplitudes of each subsystem. Therefore, using the linearized rolling process 
TABLE 1: Parameters of mill structure, rolling process, and strip material.

\begin{tabular}{lccccccc}
\hline$m_{1}(\mathrm{~kg})$ & $m_{2}(\mathrm{~kg})$ & $J_{M}\left(\mathrm{~kg} \cdot \mathrm{m}^{2}\right)$ & $k_{1}\left(\mathrm{~N} \cdot \mathrm{m}^{-1}\right)$ & $k_{2}\left(\mathrm{~N} \cdot \mathrm{m}^{-1}\right)$ & $k_{t}\left(\mathrm{~N} \cdot \mathrm{m} \cdot \mathrm{rad}^{-1}\right)$ & $c_{1}\left(\mathrm{~N} \cdot \mathrm{s} \cdot \mathrm{m}^{-1}\right)$ & $c_{2}\left(\mathrm{~N} \cdot \mathrm{s} \cdot \mathrm{m}^{-1}\right)$ \\
\hline 9200 & 203200 & 1381 & $1 \times 10^{9}$ & $6.9 \times 10^{10}$ & $7.9 \times 10^{6}$ & $5 \times 10^{4}$ & $1.64 \times 10^{7}$ \\
\hline$c_{t}\left(\mathrm{~N} \cdot \mathrm{m} \cdot \mathrm{s} \cdot \mathrm{rad}^{-1}\right)$ & $R(\mathrm{~m})$ & $L_{0}(\mathrm{~m})$ & $L_{1}(\mathrm{~m})$ & $E_{1,2}(\mathrm{GPa})$ & $u_{2}$ & $h_{0}(\mathrm{~mm})$ & 0.789 \\
\hline 4178 & 0.3 & 4.75 & 4.75 & 210 & 0.3 & $h_{1}(\mathrm{~mm})$ \\
\hline$B(\mathrm{~mm})$ & $\sigma_{0}(\mathrm{MPa})$ & $\sigma_{1}(\mathrm{MPa})$ & $\mu_{m}$ & $\bar{v}_{r}(\mathrm{~m} \cdot \mathrm{s})$ & $H(\mathrm{~mm})$ & $A(\mathrm{MPa})$ & $n$ \\
\hline 1206 & 180 & 189 & 0.04 & 19 & 2 & 810 & 0.29 \\
\hline
\end{tabular}

TABLE 2: Characteristic roots for the neighbor parameters of the instability threshold.

\begin{tabular}{|c|c|c|c|c|c|}
\hline $\bar{v}_{r}\left(\mathrm{~m} \cdot \mathrm{s}^{-1}\right)$ & $\lambda_{1,2}$ & $\lambda_{3,4}$ & $\lambda_{5}$ & $\lambda_{6,7}$ & $\lambda_{8}$ \\
\hline 20 & $-4.38 \pm 839.64 i$ & $-3.19 \pm 329.87 i$ & -275.30 & $-2.45 \pm 75.39 i$ & -120.86 \\
\hline 21 & $2.09 \pm 841.36 i$ & $-3.18 \pm 329.85 i$ & -288.59 & $-2.39 \pm 75.39 i$ & -126.61 \\
\hline
\end{tabular}

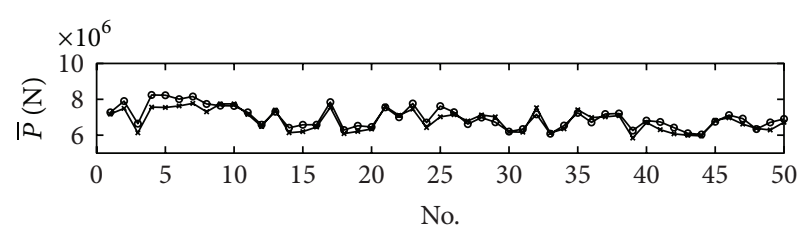

$\rightarrow$ Actual rolling force

$\rightarrow$ Calculated rolling force

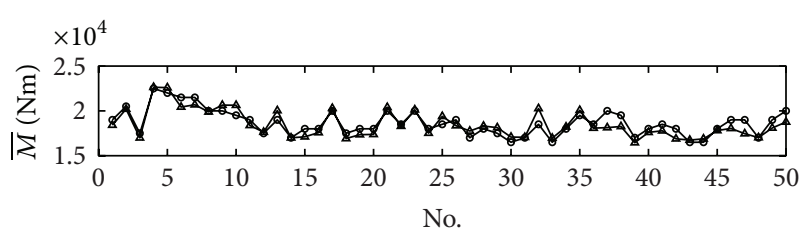

$\rightarrow$ Actual rolling torque

$\triangle$ Calculated rolling torque

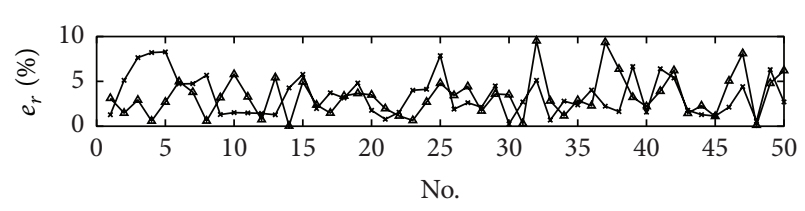

$\rightarrow$ Relative error of rolling force

$\triangle$ Relative error of rolling torque

FIGURE 5: Model validation through comparing calculated results with experimental data.

model to establish the mathematical model of mill vibration not only can ensure the accuracy but also can facilitate the stability analysis of the system.

\section{Stability Analysis and Dynamic Response}

\subsection{System Critical Rolling Speed}

5.1.1. Calculation of Critical Rolling Speed. The necessary and sufficient condition for a linear time-invariant system to be stable is that all the eigenvalues of the system or the roots of the characteristic equation possess negative real parts. As shown in (55), the characteristic matrix of the mathematical
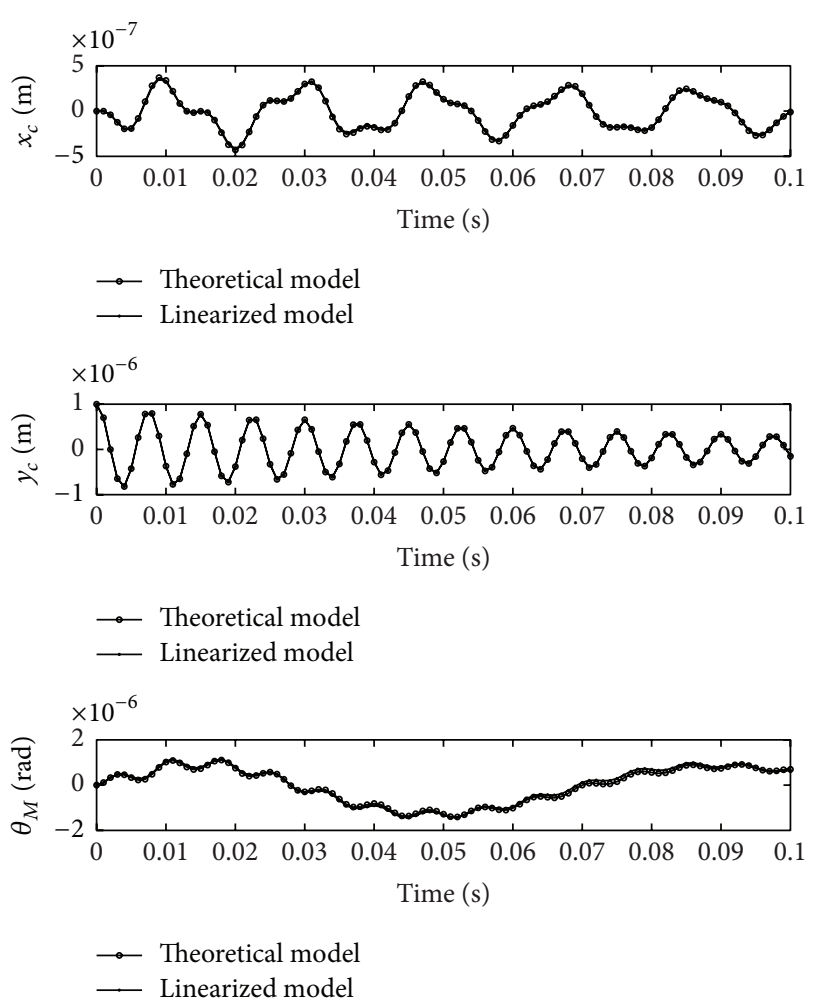

FIGURE 6: Accuracy verification of linearized dynamic rolling process model.

model is an eight-order square, and the elements of the characteristic matrix are generally represented by complex mathematical relationships of rolling process parameters. Thus it is difficult to achieve the eigenvalues of the system in a symbolic or analytical form. However, it is rather easy to calculate the eigenvalues by using a numerical method and then to determine the system stability. The calculation flow chart is shown in Figure 7.

With parameters provided in Table 1, the critical rolling speed can be calculated as $\bar{v}_{r c}=20.675 \mathrm{~m} \cdot \mathrm{s}^{-1}$. For $\bar{v}_{r}=$ $20 \mathrm{~m} \cdot \mathrm{s}^{-1}$ and $\bar{v}_{r}=21 \mathrm{~m} \cdot \mathrm{s}^{-1}$, the eigenvalues of the system are calculated, respectively, and compared in Table 2. Evidently, 
TABLE 3: Comparisons of calculated critical rolling speeds with test data.

\begin{tabular}{lccccccccccc}
\hline Number & $H(\mathrm{~mm})$ & $h_{0}(\mathrm{~mm})$ & $h_{1}(\mathrm{~mm})$ & $B(\mathrm{~mm})$ & $\sigma_{0}(\mathrm{MPa})$ & $\sigma_{1}(\mathrm{MPa})$ & $A(\mathrm{MPa})$ & $R(\mathrm{~m})$ & \multicolumn{2}{c}{ Critical rolling speed $(\mathrm{m} / \mathrm{min})$} \\
Test & Calculated & Relative error \\
\hline 1 & 2.000 & 0.789 & 0.577 & 1206 & 180 & 189 & 810 & 0.30 & 1090 & 1240 & $13.76 \%$ \\
2 & 1.974 & 0.719 & 0.512 & 1018 & 180 & 190 & 845 & 0.30 & 1229 & 1263 \\
3 & 1.962 & 0.658 & 0.472 & 1006 & 150 & 160 & 810 & 0.30 & 1271 & 1287 & $1.26 \%$ \\
4 & 1.845 & 0.645 & 0.468 & 1006 & 150 & 160 & 810 & 0.30 & 1274 & 1300 & $2.04 \%$ \\
5 & 2.022 & 0.659 & 0.468 & 1006 & 150 & 160 & 810 & 0.30 & 1274 & 1277 \\
6 & 1.966 & 0.720 & 0.513 & 1018 & 180 & 190 & 840 & 0.30 & 1156 & 1271 & $9.24 \%$ \\
7 & 1.966 & 0.720 & 0.513 & 1018 & 180 & 190 & 810 & 0.30 & 1059 & 1206 \\
8 & 2.000 & 0.755 & 0.568 & 1206 & 156 & 197 & 810 & 0.30 & 1220 & 1315 \\
9 & 1.998 & 0.754 & 0.575 & 1206 & 160 & 200 & 820 & 0.29 & 1188 & 1329 & $7.79 \%$ \\
10 & 1.964 & 0.718 & 0.517 & 1006 & 176 & 119 & 830 & 0.30 & 1300 & 1305 & $11.87 \%$ \\
\hline
\end{tabular}

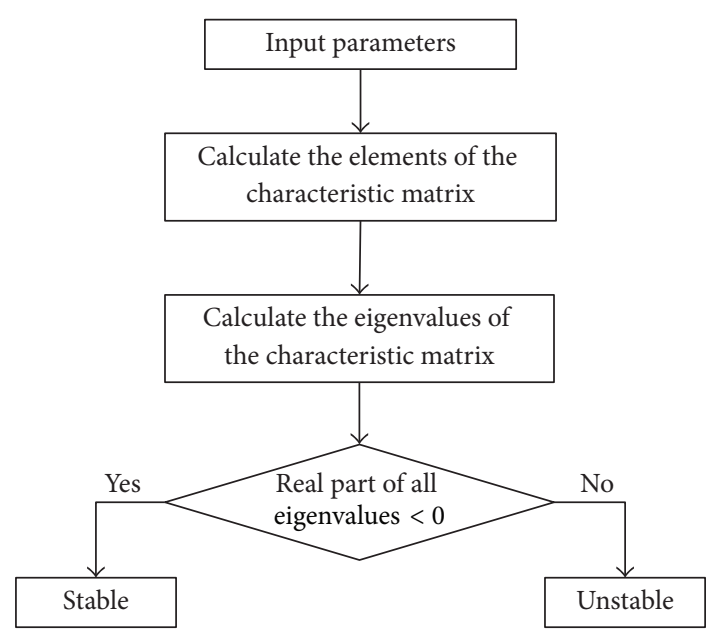

FIGURE 7: Calculation flow chart of critical rolling speed.

when the rolling speed is higher than the critical value, one pair of characteristic roots have positive real parts, and the system lost stability accordingly.

5.1.2. Comparison of Calculated Results with Test Data. In order to verify the correctness of the proposed model, the comparisons of calculated critical rolling speeds with test data are carried out. The test data are also taken from the 4 th stand of a 2030 five-stand tandem cold mill. In the vibration test, there were thirteen vibration phenomena have been observed. Here, selecting ten sets of data as examples, which are corresponding to relatively strong vibration phenomena, the comparisons are listed in Table 3. It can be seen that the calculated critical rolling speeds are very close to test values, where all relative errors are less than $14 \%$. Therefore, the proposed rolling mill multiple-modal-coupling model is accurate. Moreover, through further observation of Table 3, it can be found that all the calculated values are greater than the test values. It is because the content of this study is the vibration characteristic of a single stand mill. But the interaction between adjacent stands in tandem rolling mills can also worsen the stability of the system. Therefore, it is reasonable for the calculated value to be relatively larger.

\subsection{Dynamic Response of System Coupling Vibration}

5.2.1. Dynamic Response to Initial Disturbance of Roll Rotational Angle. For an initial disturbance $\theta_{M 0}=10^{-5} \mathrm{rad}$, the simulations on the conditions of $\bar{v}_{r}=20 \mathrm{~m} / \mathrm{s}$ and $\bar{v}_{r}=21 \mathrm{~m} / \mathrm{s}$ are carried out, and the results are shown in Figures 8 and 9.

As shown in Figures 8 and 9, although the initial disturbance is only acting on the torsional subsystem, the vibration occurs on the whole mill system. In the time-history diagrams, it is found that the system is stable when $\bar{v}_{r}<\bar{v}_{r c}$. Conversely, the system is unstable when $\bar{v}_{r}>\bar{v}_{r c}$. From the frequency spectrums, it is found that the initial roll rotational angle not only causes the vibration of each subsystem containing the frequency component of torsional vibration modal $(12.21 \mathrm{~Hz})$ but also motivates the free vibration of vertical subsystem and horizontal subsystem with their own modal frequency $(133.5 \mathrm{~Hz}$ and $52.64 \mathrm{~Hz}$, resp.). And the frequency of $133.5 \mathrm{~Hz}$ is among the frequency domain of third-octave-mode chatter. Once the system is unstable, the energy of this frequency component will increase and extend sharply in the mill system.

5.2.2. Dynamic Response to Periodic Disturbance of Strip Entry Thickness. Assuming that the variation of strip entry thickness is $h_{0 \mathrm{var}}=10^{-6} \times \sin (200 \cdot t)$ and without any other initial disturbance, the dynamic responses on the conditions of $\bar{v}_{r}=20 \mathrm{~m} / \mathrm{s}$ and $\bar{v}_{r}=21 \mathrm{~m} / \mathrm{s}$ are analyzed and shown in Figures 10 and 11 .

Likewise, the system is stable when $\bar{v}_{r}<\bar{v}_{r c}$ and unstable when $\bar{v}_{r}>\bar{v}_{r c}$. Since the periodic fluctuation of the strip entry thickness is equivalent to introducing a periodic excitation into the system, the vibration frequencies of each subsystem are all close to $32 \mathrm{~Hz}$ (the frequency of excitation) when the system is stable. However, when the system lost stability, the energy of the excitation frequency component is almost unchanged, but the frequency component, which belongs to the frequency domain of third-octave-mode chatter, would rise and spread rapidly in the system. 

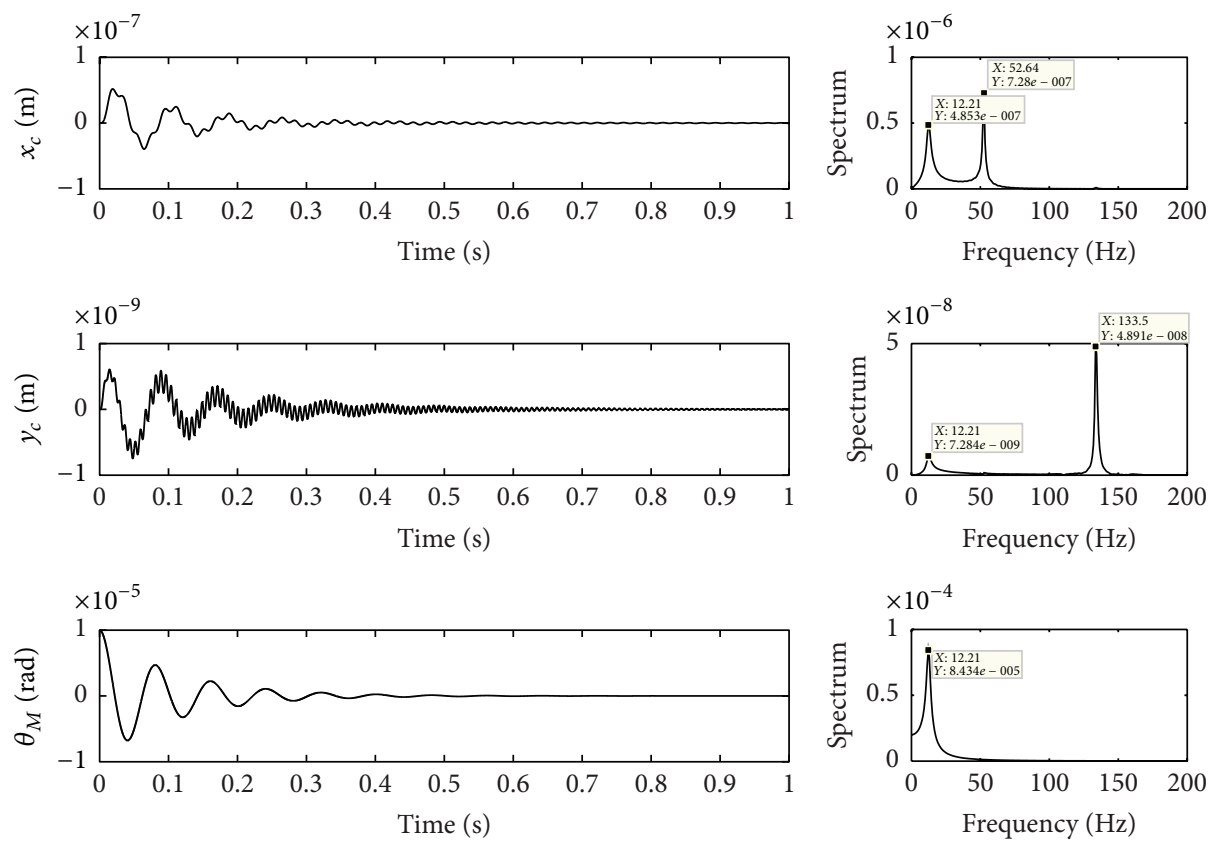

FIGURE 8: Dynamic response to the variation in roll rotational angle for $\bar{v}_{r}=20 \mathrm{~m} \cdot \mathrm{s}^{-1}$.
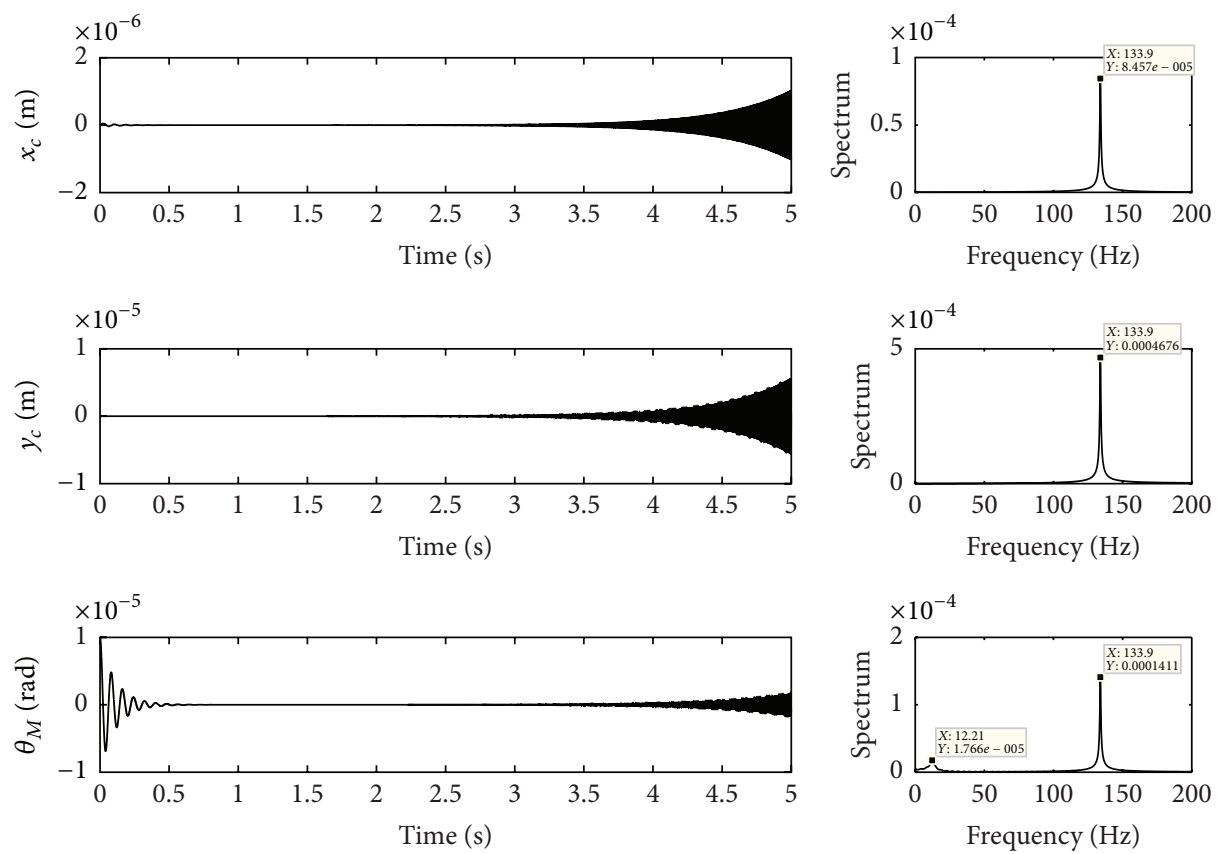

FIGURE 9: Dynamic response to the variation in roll rotational angle for $\bar{v}_{r}=21 \mathrm{~m} \cdot \mathrm{s}^{-1}$.

\section{Influence of Parameters on System Stability}

\subsection{Rolling Process Parameters}

6.1.1. Tension at Entry and Exit. With parameters provided in Table 1, the influences of tensions at entry and exit on the system stability are depicted in Figure 12. As shown in Figure 12(a), with the increase of tensions at entry and exit, the critical rolling speed decreases gradually. The reason is that larger tensions correspond to the smaller rolling stiffness
$\left(a_{F_{y}}, y_{c}\right)$. Thus the stability of the system is weakened. Through further comparison, it can be seen that the influence of the entry tension is greater than the exit tension. Figure 12(b) is the change of the critical rolling speed under the coupling effect of entry tension and exit tension. Figure 12(c) is the critical rolling speed contour in the coupling parameters plane $\left(\sigma_{1}, \sigma_{0}\right)$. It can be seen from Figures 12(b) and 12(c) that the influences of tensions at entry and exit on the system stability are approximately linear. 

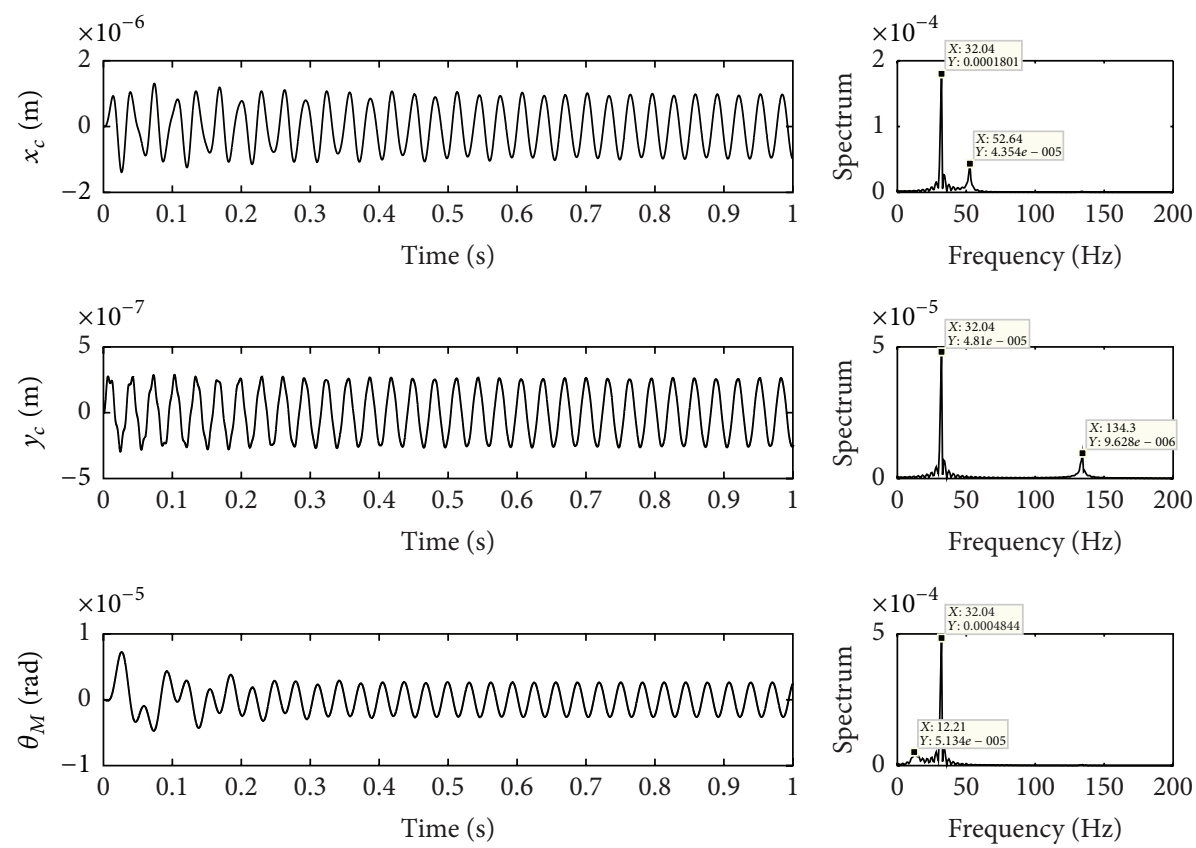

FIGURE 10: Dynamic response to periodic disturbance of strip entry thickness for $\bar{v}_{r}=20 \mathrm{~m} / \mathrm{s}$.
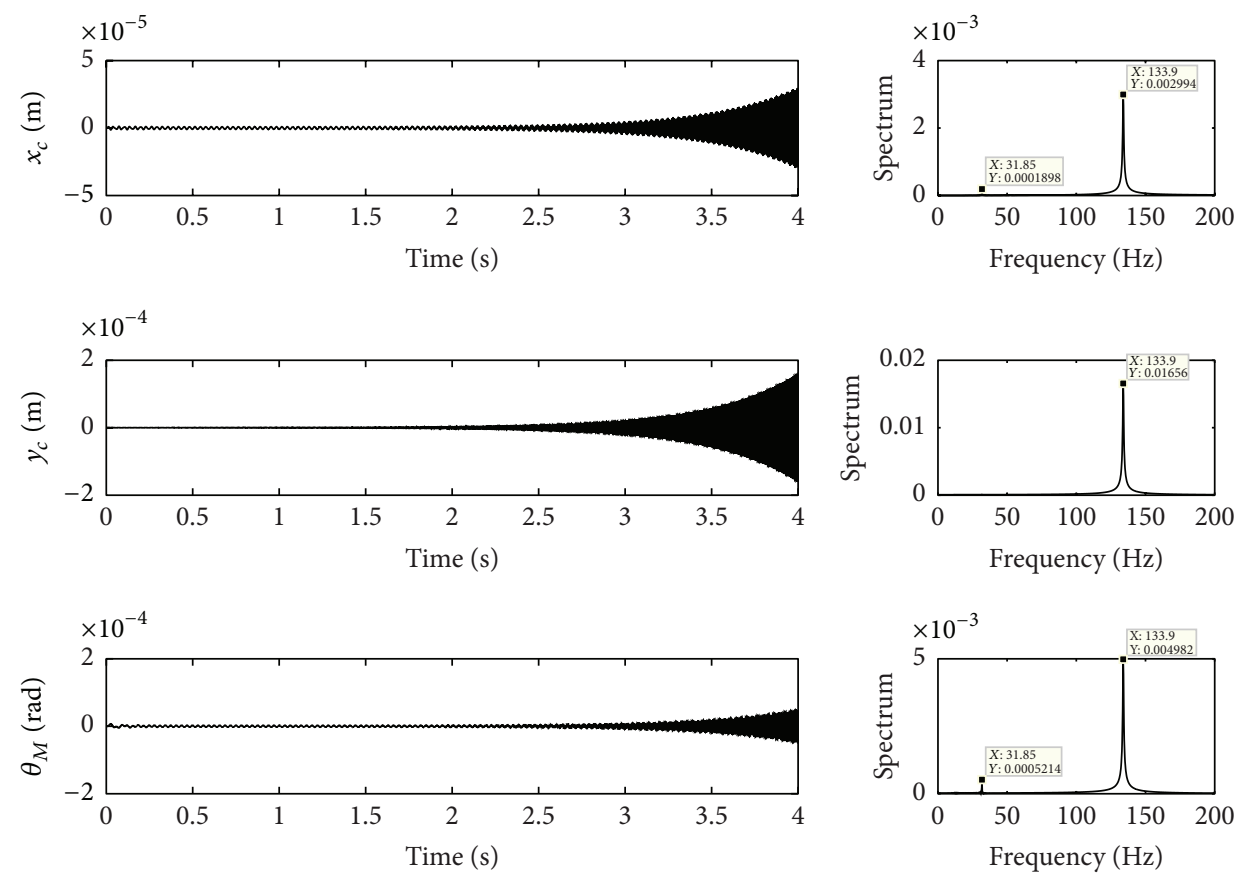

FIGURE 11: Dynamic response to periodic disturbance of strip entry thickness for $\bar{v}_{r}=21 \mathrm{~m} / \mathrm{s}$.

6.1.2. Reduction Ratio. Similarly, with parameters provided in Table 1, the change of the critical rolling speed with different reduction ratios is plotted in Figure 13. In this section, the conditions of invariable entry thickness and invariable exit thickness are considered, respectively. In Figure 13, the two curves almost overlap. And both conditions show that the increasing reduction ratio can result in lower system stability. The reason is that the larger reduction ratio means greater rolling force. So, the fluctuation of the rolling force will increase under the same disturbance, and the emergence of vibration happens more easily.

6.1.3. Friction Coefficient and Strip Width. Figure 14 represents the influences of friction coefficient and strip width on system stability. From Figure 14(a), it can be seen that the critical rolling speed increases significantly with the increase 


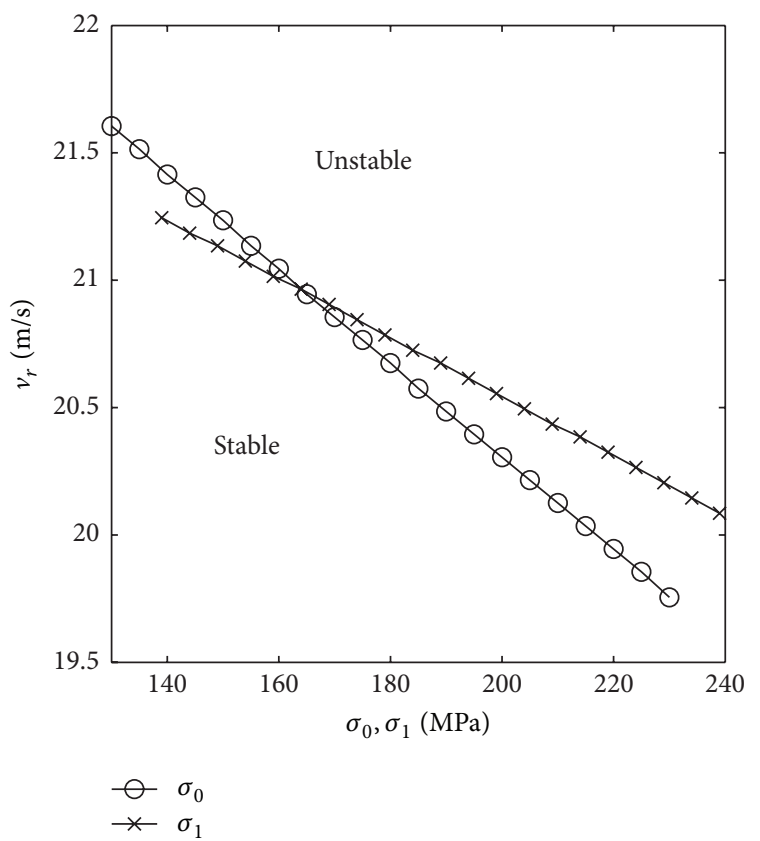

(a)

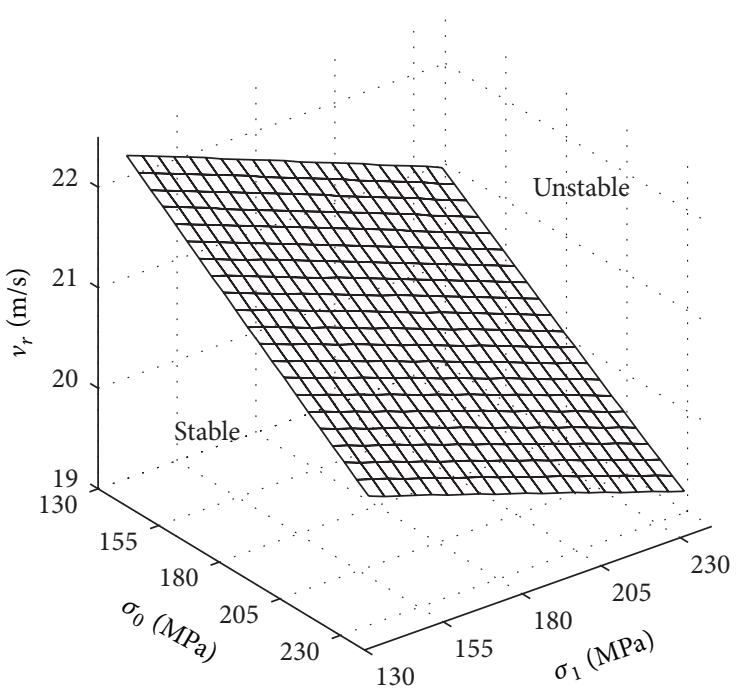

(b)

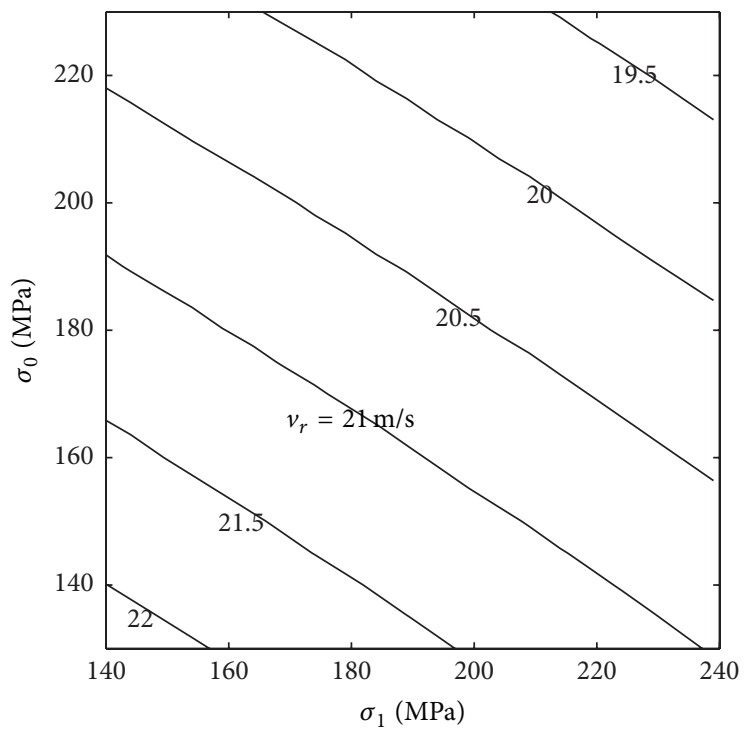

(c)

FIGURE 12: Influences of tensions at entry and exit on system stability.

of the friction coefficient. The reason is that the friction in the roll gap acts as a positive damping in the system. As Figure 14(b) displays, the wider the strip is, the more stable the system will be. It is because the wider strip corresponds to relatively larger rolling contact stiffness and rolling damping, which can strengthen the system stability.

6.1.4. Strip Material Parameters. Figures 15(a) and 15(b) depict that the critical rolling speed increases with the increase of material parameter $A$ and increases with the decrease of material parameter $n$. Equation (25) indicates that the mean deformation resistance $K$ is an increasing function of $A$ and is a decreasing function of $n$. Therefore, with the increase of the mean deformation resistance $K$, the critical rolling speed increases monotonically. The reason is that the larger mean deformation resistance signifies the greater stiffness of the system. Naturally, the stability of the system will be strengthened.

From Figure 15(c), increasing Young's modulus of the strip leads to destabilization of the system. It is because the increasing Young modulus means larger variations of tensions at entry and exit. In addition, the steep slope of the curve indicates a high sensitivity of the system stability to Young's modulus of the strip. 


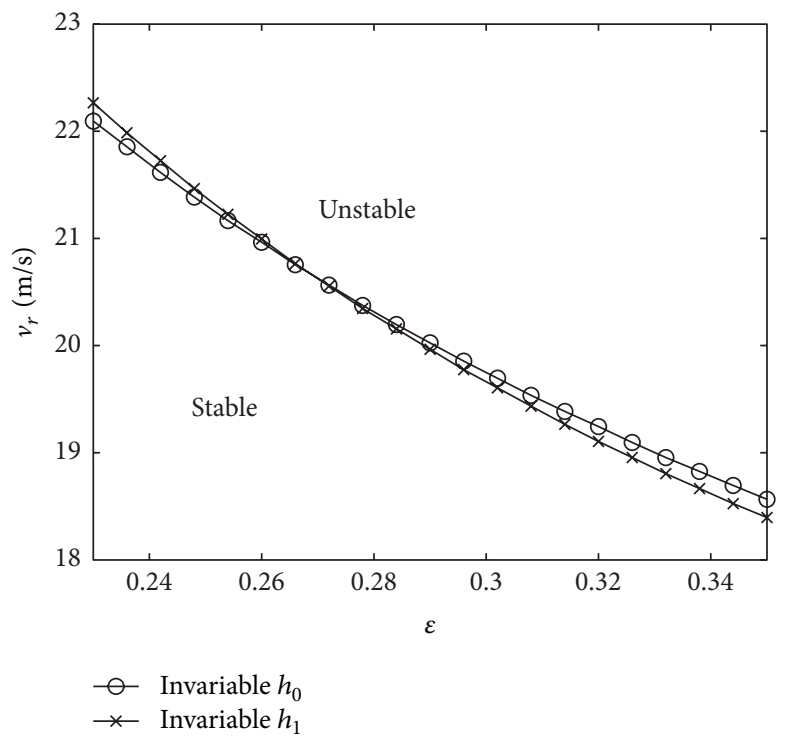

FIGURE 13: Influence of reduction ratio on system stability.

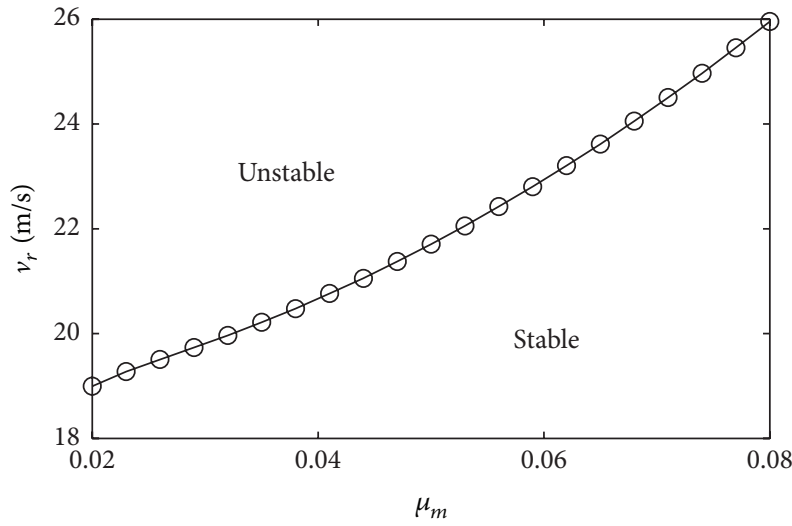

(a)

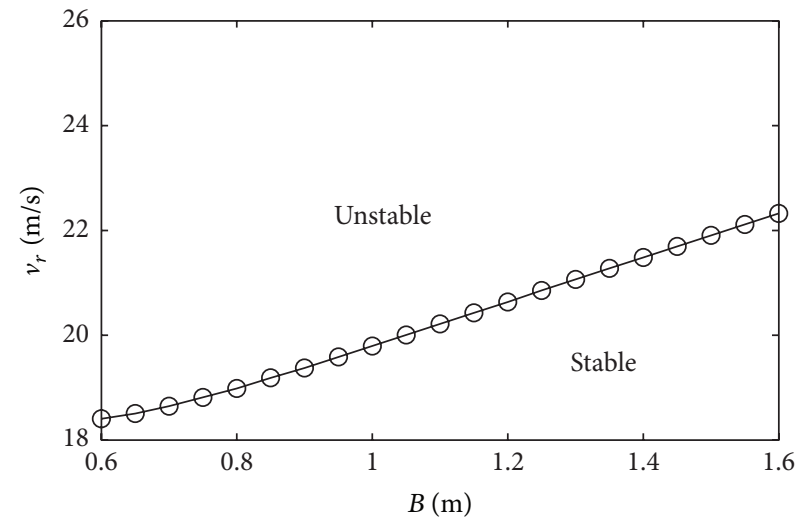

(b)

FIGURE 14: Influences of friction coefficient and strip width on system stability.

6.2. Mill Structure Parameters. As the vertical-torsionalhorizontal coupled dynamic model is established by associating the rolling process model with the structure model, so mill structure parameters also influence the system stability. The impact trend of each structure parameter on the system stability is shown in Figures 16-19. And the impact extents of process parameters and structure parameters will be compared in Section 6.4.

As shown in Figures 16 and 18, the structure parameters of horizontal subsystem and torsional subsystem almost have no effect on the critical rolling speed. However, the vertical subsystem structure parameters have a significant impact on the stability of the system (Figure 17). It is because the thirdoctave-mode chatter mainly reflects in the vertical subsystem.

From Figure 19(a), it can be seen that the critical rolling speed will increase with the increase of the work roll radius. It is because the increase of the work roll radius means increasing the contact arc length, which will greaten the friction effect of the roll gap and then enhance the system stability. In Figures 19(b) and 19(c), the critical rolling speed increases with the increase of $L_{0}$ and $L_{1}$. The reason is that the larger distances $L_{0}$ and $L_{1}$ will reduce the variations of tensions and then strengthen the system stability. Since the effect of the entry tension is greater than the exit tension, the effect of $L_{0}$ is relatively larger.

6.3. Experimental Verification. As rolling mill vibration will affect the normal production and even lead to equipment failure, therefore, it is not easy to conduct an all-round rolling mill vibration test to verify the influences of all aforementioned parameters on the system stability. In this section, a series of experiments, which are corresponding to the friction coefficient, the reduction ratio, and the exit tension, respectively, are used to verify the above relevant conclusions. Here, the experiment used to verify the influence of the friction coefficient is taken from the field vibration test of the 4th stand of a 2030 five-stand tandem cold mill. The experiments used to verify the influences of the reduction 


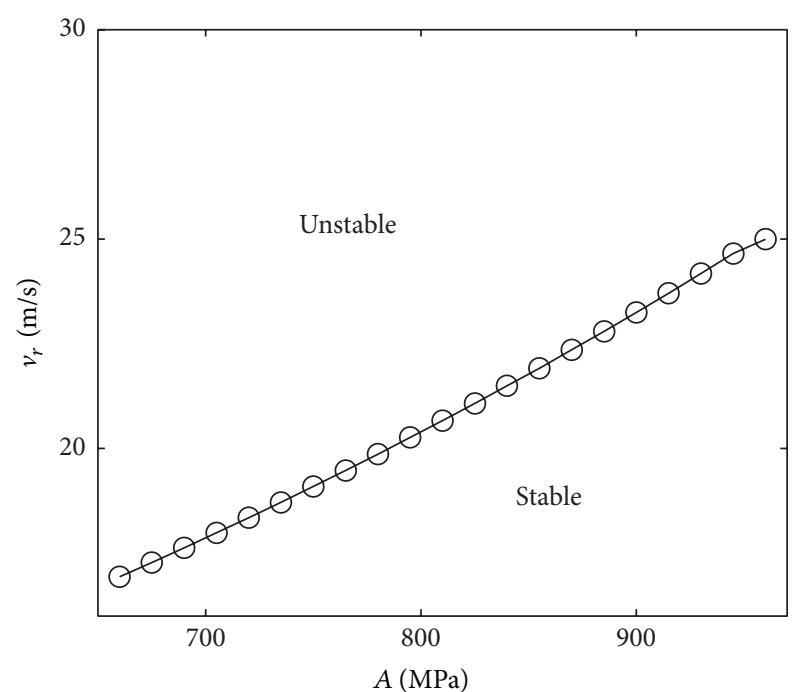

(a)

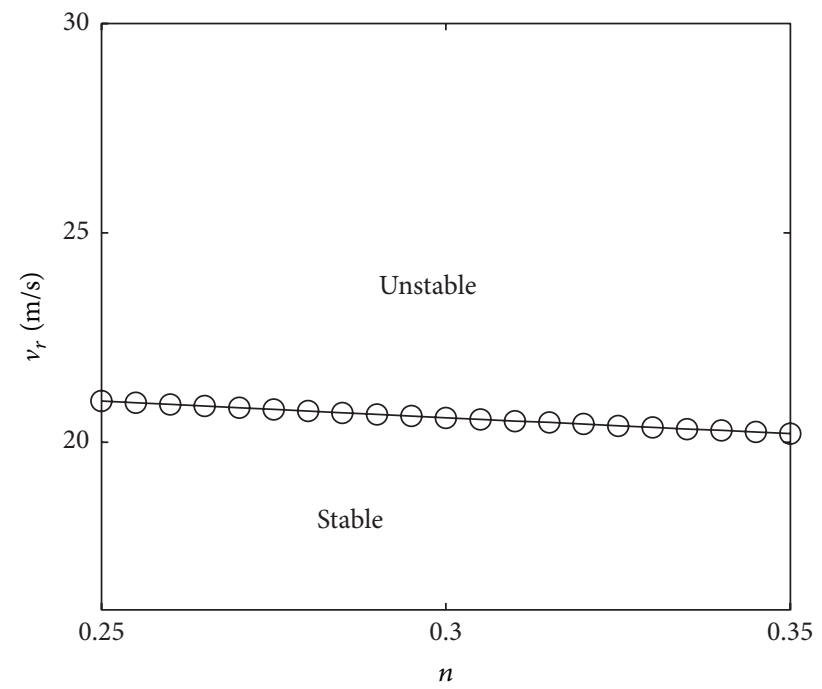

(b)

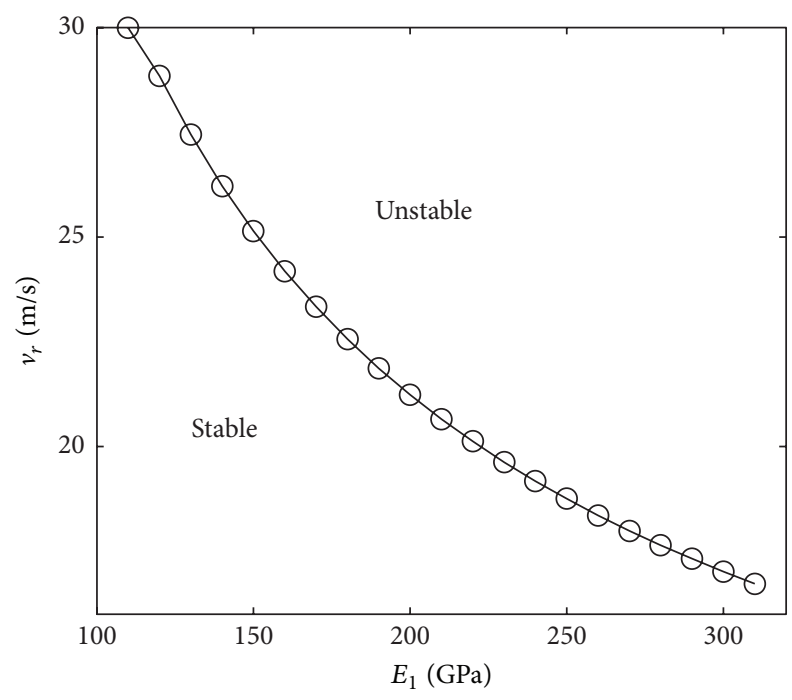

(c)

FIGURE 15: Influences of strip material parameters on system stability.

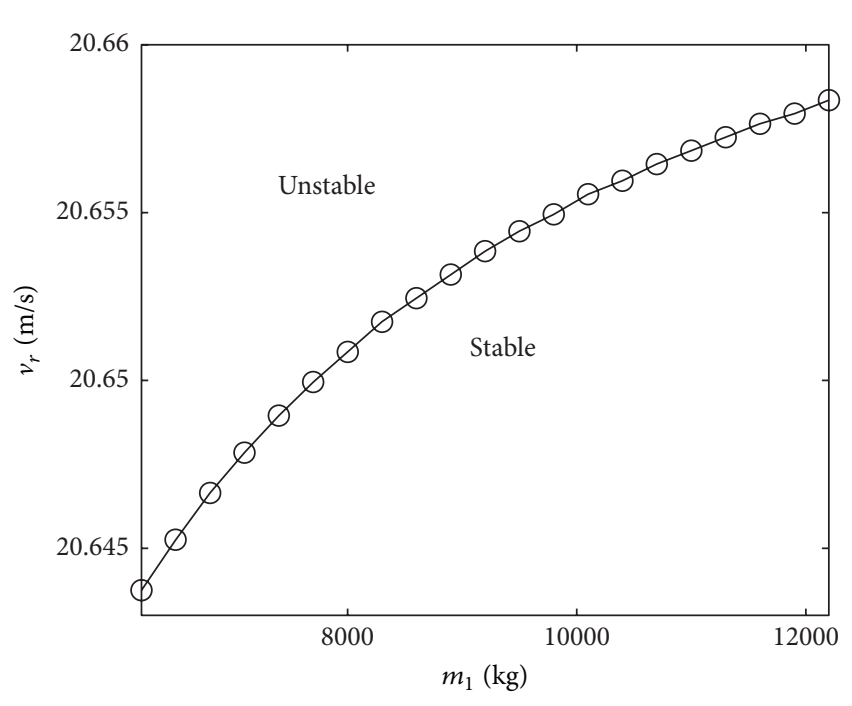

(a)

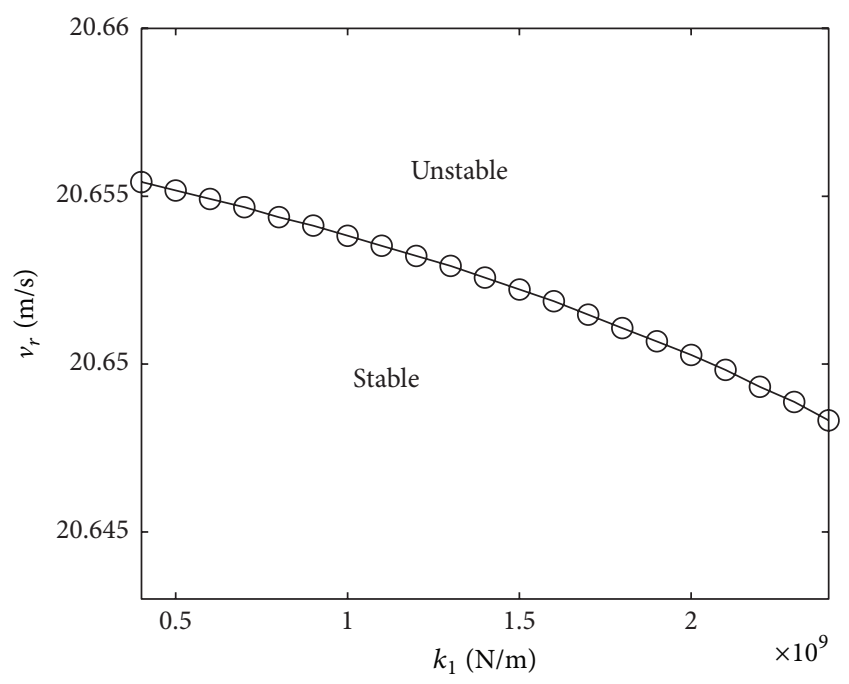

(b)

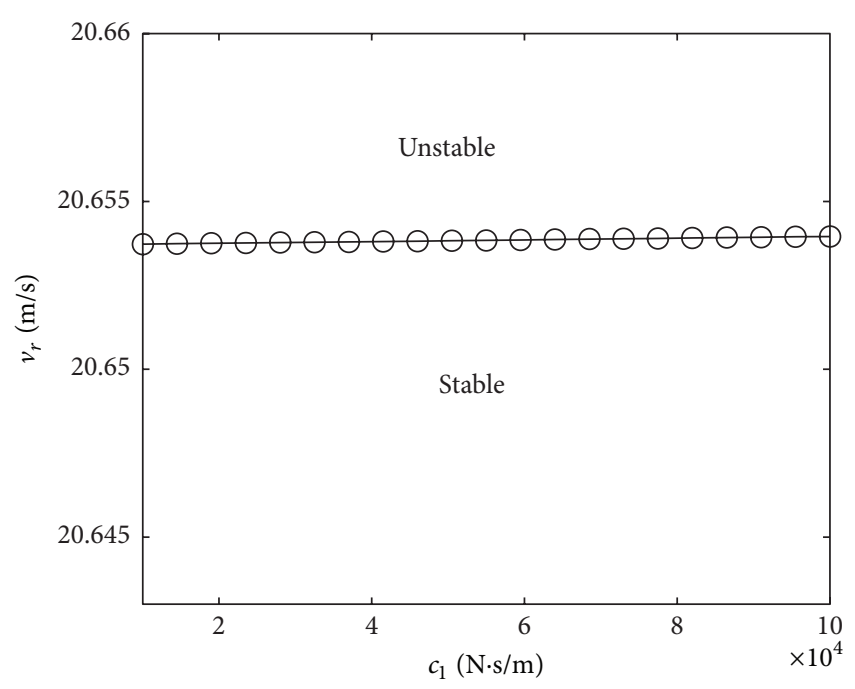

(c)

FIGURE 16: Influences of horizontal structural parameters on system stability. 


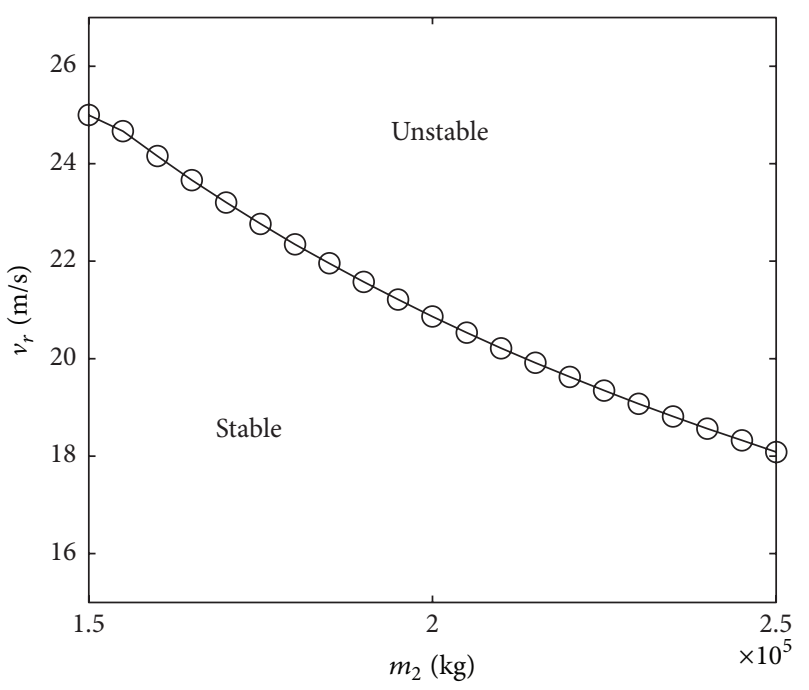

(a)

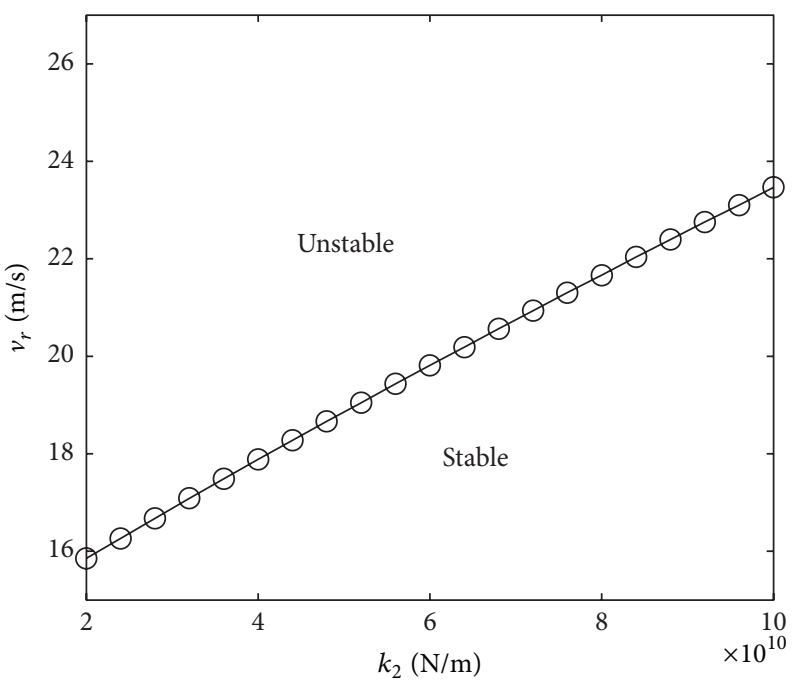

(b)

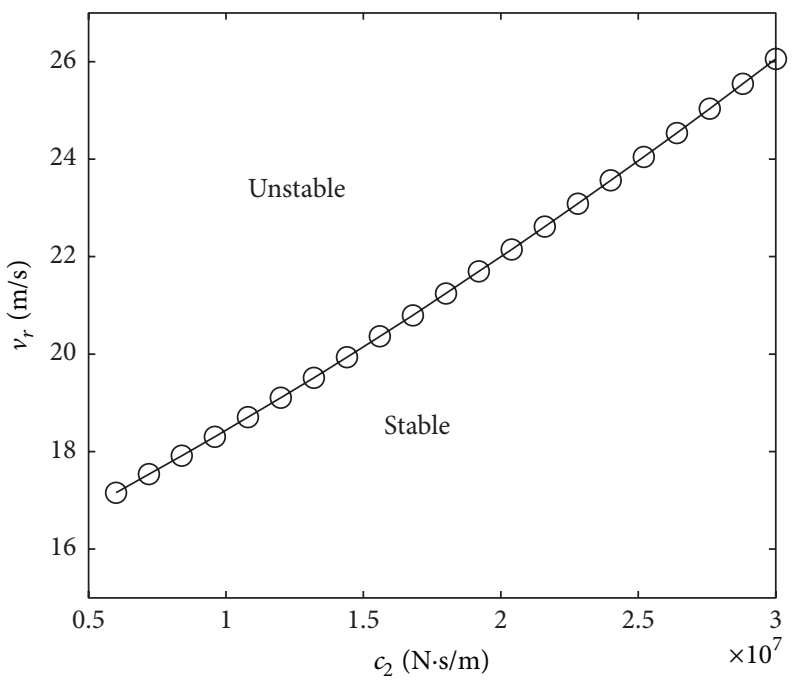

(c)

FIGURE 17: Influences of vertical structural parameters on system stability.

ratio and the exit tension are performed on a laboratory test rolling mill.

In order to observe the influence of the friction coefficient on the system stability, rolling lubricants of different types and different concentrations are applied to the 2030 five-stand tandem cold mill system in the field test, as shown in Table 4. For this rolling mill system, the original rolling lubricant type is N54. In that case, the system would lose its stability when the rolling speed was raised to $1250 \mathrm{~m} / \mathrm{min}$. Then, the rolling lubricant was changed to N428, where lubricating property is better than N54. As a result, the system was unstable when the rolling speed was only raised to $1000 \mathrm{~m} / \mathrm{min}$. Thus, the system is more prone to vibration under the condition of the smaller friction coefficient. The same conclusion can also be obtained by the experiment of rolling lubricants with different concentrations. As shown in Table 4, for the same type of rolling lubricant, the lower the concentration of the emulsion is (scilicet, the friction coefficient is larger), the more stable the system will be. Obviously, these experimental results are consistent with the analysis conclusion mentioned in Section 6.1.3.

As the 2030 five-stand tandem cold mill system is highly automatic, it is difficult for artificial participation. Therefore, in the field test, the influences of other parameters on the system stability are not investigated. In the following, the laboratory test rolling mill is used to verify some of other parameters' influence. Since it is not possible to generate vibration on the laboratory-scale rolling mill, hence, in the experiments, these influences can only be verified indirectly through comparing the vibration intensities of the system under different parameters.

Figure 20 shows the dynamic rolling experiment under different reduction ratios. In this experiment, the rolling speed is $0.17 \mathrm{~m} / \mathrm{s}$, and the tensile forces of the strip at entry and exit are $8000 \mathrm{~N}$ and $4000 \mathrm{~N}$, respectively. As shown in Figure 20, the reduction ratio in Region II is larger than that 


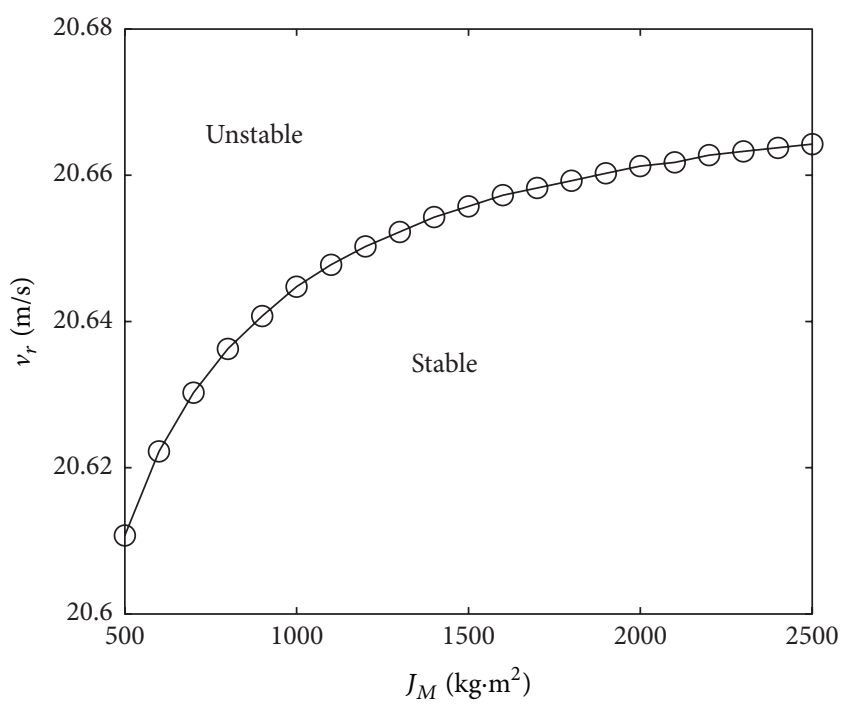

(a)

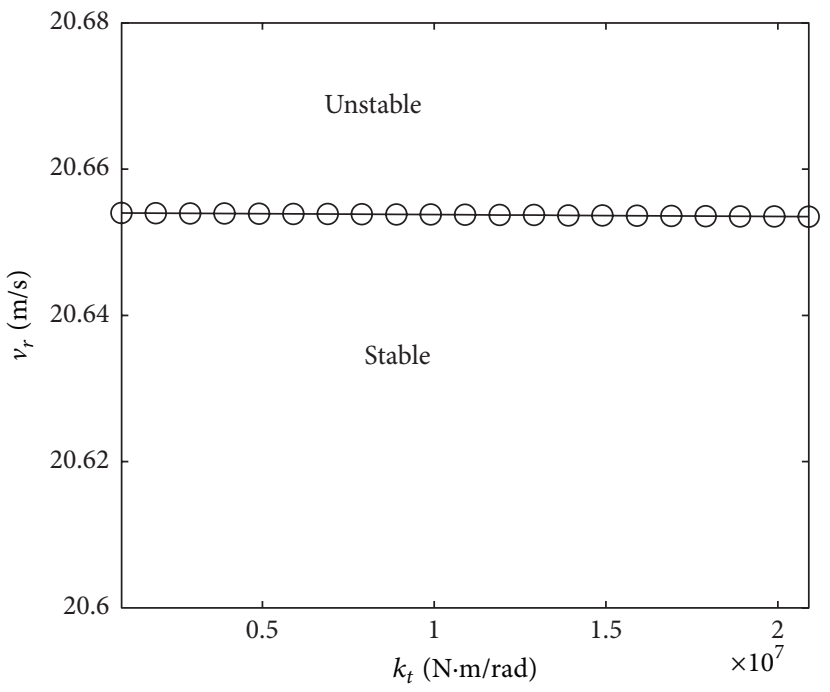

(b)

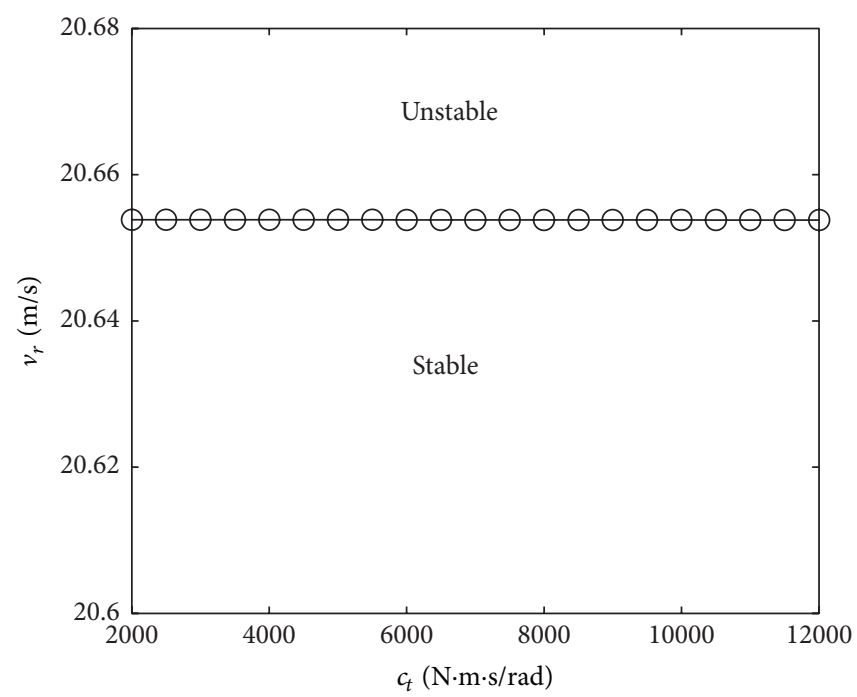

(c)

FIGURE 18: Influences of torsional structural parameters on system stability.

in Region I, and the system vibration intensity in Region II is also relatively strong. Therefore, it can be inferred that the system is more prone to vibration with larger reduction ratio. This conclusion is consistent with the analysis in Section 6.1.2.

Figure 21 shows the dynamic rolling experiment under different exit tensions and different rolling speeds. In this experiment, the entry tensile force is $10000 \mathrm{~N}$, and the reduction ratio is kept unchanged. As the rolling speed in Region II is larger than that in Region I, the system vibration intensity is also relatively strong, while, in Region IV, even though the rolling speed is smaller than that in Region II, the system vibration intensity is stronger. It is because the exit tension in that region is larger than Region II. This experiment result confirmed the analysis conclusion in Section 6.1.1. Scilicet, the larger tensions will weaken the system stability. In Region III, as both the rolling speed and the exit tension are larger than other regions, the system vibration intensity became strong significantly.

6.4. Sensitivity Analysis of Parameters on the System Stability. In order to compare the impact of parameters on the system stability, a dimensionless relative sensitivity factor is defined as follows:

$$
\begin{aligned}
& S(u)=\max \left\{\left|S_{i}(u)\right|\right\} ; \\
& S_{i}(u)=\frac{\left(v_{r c, i+1}-v_{r c, i}\right) / v_{r c, i}}{\left(u_{i+1}-u_{i}\right) / u_{i}} \\
& \quad(i=1,2, \ldots) .
\end{aligned}
$$

The relative sensitivity factors for aforementioned parameters are given in Table 5, in which the negative sign denotes 


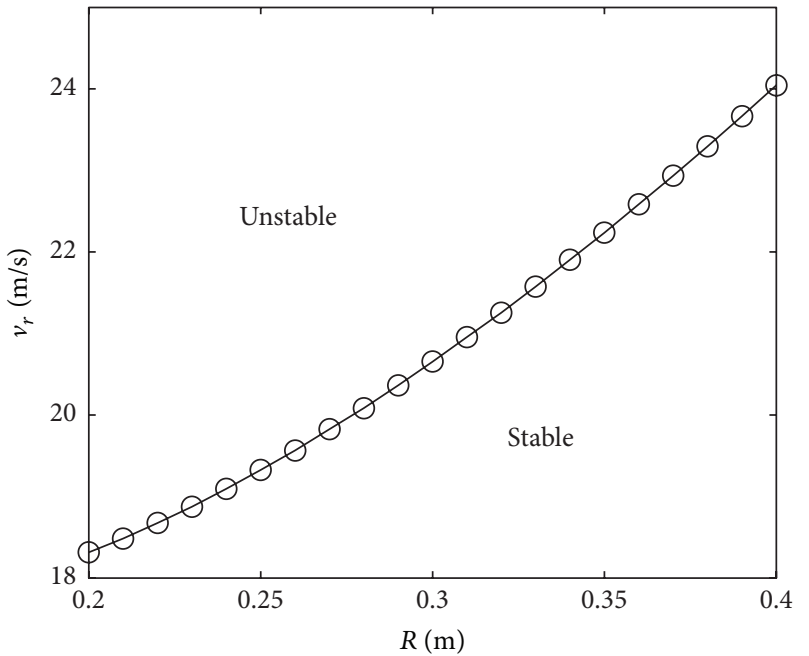

(a)

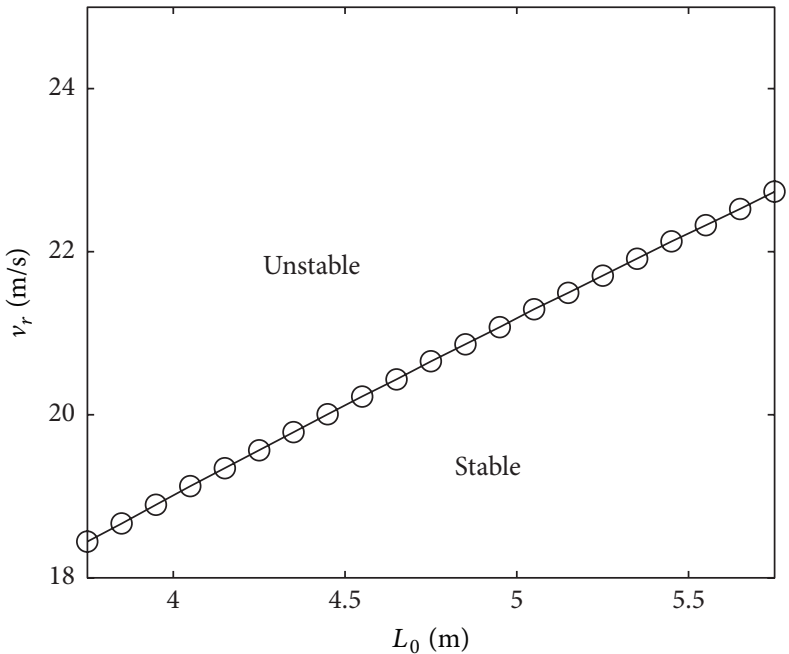

(b)

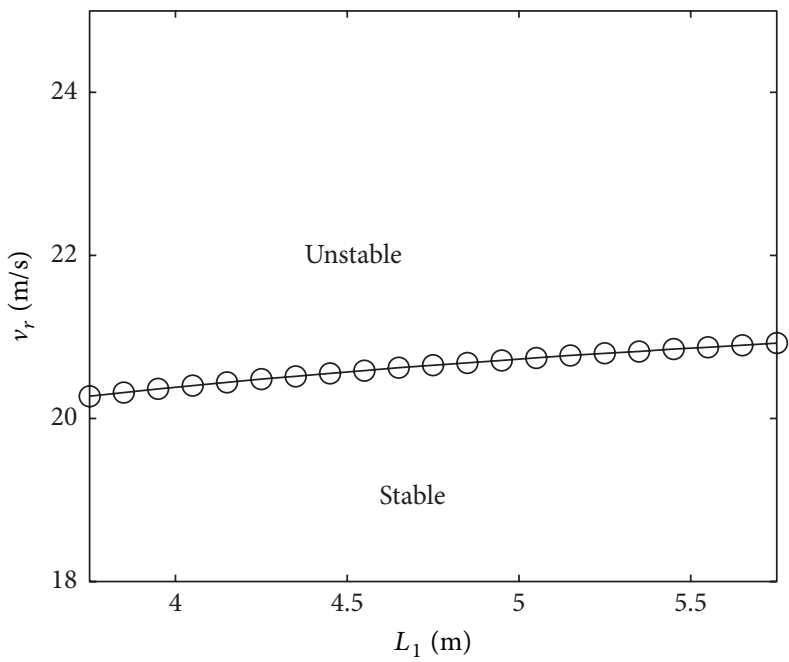

(c)

FIGURE 19: Influences of work roll radius and distances between adjacent stands on system stability.

TABLE 4: Influence of rolling lubricants on system stability.

\begin{tabular}{|c|c|c|c|c|}
\hline \multicolumn{5}{|c|}{ Rolling specification: $2 \rightarrow 0.5(\mathrm{~mm})$} \\
\hline \multirow{2}{*}{ Rolling lubricants } & \multicolumn{2}{|c|}{ Emulsion concentration (\%) } & \multirow{2}{*}{ Rolling speed (m/min) } & \multirow{2}{*}{ System stability } \\
\hline & Tank \#3 & Tank \#5 & & \\
\hline \multirow{3}{*}{ N54 } & 2.2 & 1.2 & 1200 & Stable \\
\hline & 2.2 & 1.2 & 1250 & Unstable \\
\hline & 1.5 & 0.6 & 1480 & Stable \\
\hline \multirow{3}{*}{ N428 } & 2.2 & 1.2 & 988 & Stable \\
\hline & 2.2 & 1.2 & 1000 & Unstable \\
\hline & 1.6 & 0.7 & 1413 & Stable \\
\hline
\end{tabular}

TABLE 5: Relative sensitivity factors for the following parameters.

\begin{tabular}{|c|c|c|c|c|c|c|c|c|c|}
\hline$S\left(\sigma_{0}\right)$ & $S(\varepsilon)$ & $S(B)$ & $S(A)$ & $S\left(m_{1}\right)$ & $S\left(c_{1}\right)$ & $S\left(k_{1}\right)$ & $S\left(m_{2}\right)$ & $S\left(c_{2}\right)$ & $S\left(k_{2}\right)$ \\
\hline-0.227 & -0.458 & 0.294 & 1.231 & $1.502 \times 10^{-3}$ & $1.469 \times 10^{-5}$ & $-6.126 \times 10^{-4}$ & -0.649 & 0.479 & 0.374 \\
\hline$S\left(\sigma_{1}\right)$ & $S\left(\mu_{m}\right)$ & $S\left(E_{1}\right)$ & $S(n)$ & $S\left(J_{M}\right)$ & $S\left(c_{t}\right)$ & $S\left(k_{t}\right)$ & $S\left(L_{0}\right)$ & $S\left(L_{1}\right)$ & $S(R)$ \\
\hline-0.139 & 0.504 & -0.583 & -0.136 & 0.003 & $-4.261 \times 10^{-6}$ & $-2.891 \times 10^{-5}$ & 0.527 & 0.085 & 0.395 \\
\hline
\end{tabular}




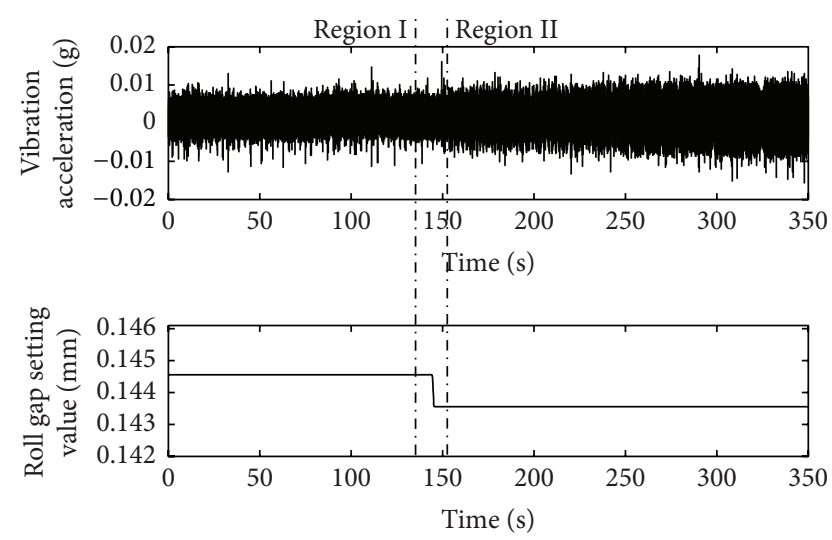

Figure 20: Dynamic rolling experiment under different reduction ratios.

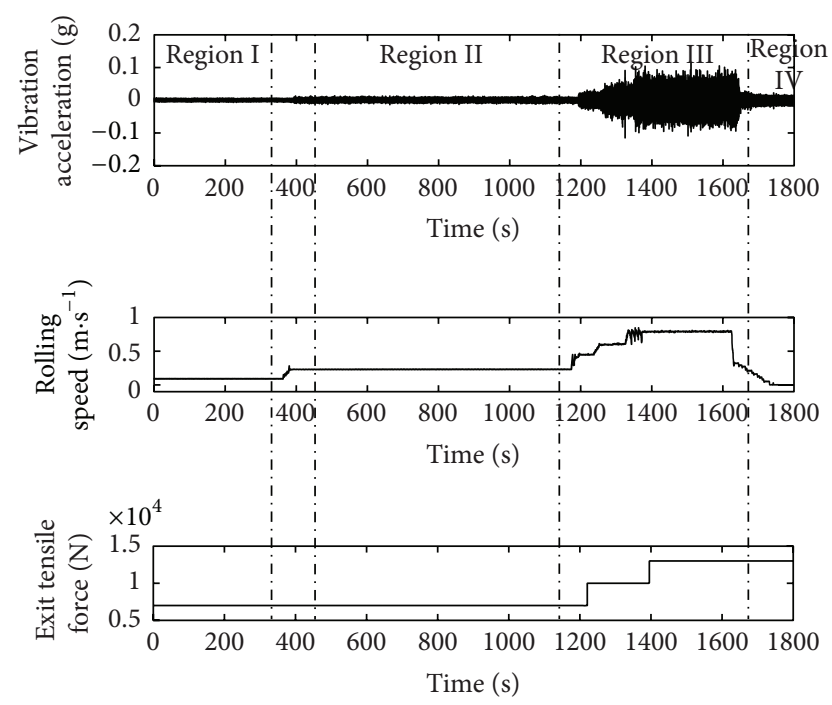

FIGURE 21: Dynamic rolling experiment under different exit tensions and different rolling speeds.

the fact that the critical rolling speed decreases with the relevant parameter increasing.

It can be seen from Table 5 that the influences of process parameters on the system stability are generally larger than structure parameters. Among structure parameters, the relative sensitivity factors of the work roll radius $R$, the distance between this stand and the upstream stand $L_{0}$, and the structure parameters of vertical subsystem are relatively large. Clearing the impact trend and extent of these parameters on the system stability can help to formulate a reasonable rolling procedure specification and achieve a reasonable structural dynamic modification strategy as well.

\section{Conclusions}

For the wide existence of vibration in cold rolling mill, this paper has conducted a systematic study on multiplemodal-coupling modeling and stability analysis of rolling mill vibration. The following conclusions are drawn:
(1) A rolling mill multiple-modal-coupling vibration model is established by coupling the rolling mill structure model and the dynamic rolling process model. In the structure model, the rolls are allowed to vibrate in vertical, horizontal, and torsional directions. And the dynamic rolling process model, in which dynamic changes of the roll gap in the state of vibration are taken into consideration, is formulated based on the Hill simplified formula of Bland-Ford rolling force model.

(2) The effectiveness of the theoretical rolling process model is verified by the test data of a cold rolling mill, and the accuracy of the linearized rolling process model is testified by the simulation analysis. This work can lay the foundation for further studying of dynamic characteristics of the mill system.

(3) Based on the proposed vertical-torsional-horizontal coupling vibration model, the critical rolling speed for system to be stable is calculated by the method of nonlinear dynamics. And the accuracy of calculated critical rolling speed is verified by experimental data. On this basis, the system dynamic responses in both time and frequency domains are analyzed under different initial disturbances. And the results are used to discuss the interaction effect of different mill subsystems.

(4) Finally, the influences of rolling process parameters and mill structure parameters on the system stability are investigated. And some experiments, which are corresponding to the friction coefficient, the reduction ratio, and the exit tension, respectively, are performed to verify the correctness of the relevant analysis conclusion. Then, the impact extents of these parameters on the system stability are compared by calculating their relative sensitivity factors. These conclusions not only can provide technical support for formulating a reasonable rolling process planning but also can lay a theoretical foundation for mill structure dynamics modification.

\section{Appendix}

\section{A. Coefficients of the Linearized Parameters in Section 3.2}

In (31)

$$
\begin{aligned}
& \left(\frac{\partial x_{0}}{\partial h_{0}}\right)_{s}=-\sqrt{\frac{R^{\prime}}{4\left(\bar{h}_{0}-\bar{h}_{c}\right)}} ; \\
& \left(\frac{\partial x_{0}}{\partial h_{c}}\right)_{s}=\sqrt{\frac{R^{\prime}}{4\left(\bar{h}_{0}-\bar{h}_{c}\right)}} ; \\
& \left(\frac{\partial x_{0}}{\partial x_{c}}\right)_{s}=1 .
\end{aligned}
$$




$$
\begin{aligned}
& \left(\frac{\partial x_{1}}{\partial h_{c}}\right)_{s}=\left(\frac{\partial x_{1}}{\partial v_{r}}\right)_{s}=\left(\frac{\partial x_{1}}{\partial x_{n}}\right)_{s}=\left(\frac{\delta x_{1}}{\delta \dot{x}_{c}}\right)_{s}=0 ; \\
& \left(\frac{\partial x_{1}}{\partial \dot{y}_{c}}\right)_{s}=-\frac{R^{\prime} \bar{h}_{c}}{\bar{v}_{r} \bar{h}_{n}} ; \\
& \left(\frac{\partial x_{1}}{\partial x_{c}}\right)_{s}=1 .
\end{aligned}
$$

$$
\begin{aligned}
& \left(\frac{\partial \alpha}{\partial h_{0}}\right)_{s}=\frac{1}{2 \sqrt{R^{\prime}\left(\bar{h}_{0}-\bar{h}_{c}\right)}} \\
& \left(\frac{\partial \alpha}{\partial h_{c}}\right)_{s}=-\frac{1}{2 \sqrt{R^{\prime}\left(\bar{h}_{0}-\bar{h}_{c}\right)}} .
\end{aligned}
$$

In (38)

In (34)

$$
\begin{aligned}
& \left(\frac{\partial x_{n}}{\partial \sigma_{0}}\right)_{s}=\frac{\bar{h}_{0}}{4 \mu_{m}\left(\bar{K}-\bar{\sigma}_{m}\right) \bar{Q}_{p}} ; \\
& \left(\frac{\partial x_{n}}{\partial \sigma_{1}}\right)_{s}=-\frac{\bar{h}_{1}}{4 \mu_{m}\left(\bar{K}-\bar{\sigma}_{m}\right) \bar{Q}_{p}} ; \\
& \left(\frac{\partial x_{n}}{\partial h_{0}}\right)_{s}=\frac{\bar{\sigma}_{0}}{4 \mu_{m}\left(\bar{K}-\bar{\sigma}_{m}\right) \bar{Q}_{p}} ; \\
& \left(\frac{\partial x_{n}}{\partial x_{c}}\right)_{s}=1 ; \\
& \left(\frac{\partial x_{n}}{\partial h_{1}}\right)_{s}=-\frac{\bar{\sigma}_{1}}{4 \mu_{m}\left(\bar{K}-\bar{\sigma}_{m}\right) \bar{Q}_{p}} ; \\
& \left(\frac{\partial x_{n}}{\partial \alpha}\right)_{s}=-\frac{R^{\prime}}{2} \cos \bar{\alpha}+\frac{R^{\prime}}{2 \mu_{m}} \sin \bar{\alpha} ; \\
& \left(\frac{\partial x_{n}}{\partial \delta}\right)_{s}=-\frac{R^{\prime}}{2} \cos \bar{\delta}-\frac{R^{\prime}}{2 \mu_{m}} \sin \bar{\delta}=-\frac{R^{\prime}}{2} \\
& \left(\frac{\partial x_{n}}{\partial K}\right)_{s}=\frac{\bar{\sigma}_{1} \bar{h}_{1}-\bar{\sigma}_{0} \bar{h}_{0}}{4 \mu_{m}\left(\bar{K}-\bar{\sigma}_{m}\right)^{2} \bar{Q}_{p}} ; \\
& \left(\frac{\partial x_{n}}{\partial \sigma_{m}}\right)_{s}=-\frac{\bar{\sigma}_{1} \bar{h}_{1}-\bar{\sigma}_{0} \bar{h}_{0}}{4 \mu_{m}\left(\bar{K}-\bar{\sigma}_{m}\right)^{2} \bar{Q}_{p}} ; \\
& \left(\frac{\partial x_{n}}{\partial Q_{p}}\right)_{s}=\frac{\bar{\sigma}_{1} \bar{h}_{1}-\bar{\sigma}_{0} \bar{h}_{0}}{4 \mu_{m}\left(\bar{K}-\bar{\sigma}_{m}\right) \bar{Q}_{p}^{2}} ;
\end{aligned}
$$

$$
\begin{aligned}
& \left(\frac{\partial \delta}{\partial x_{c}}\right)_{s}=\frac{1}{R^{\prime}} \\
& \left(\frac{\partial \delta}{\partial x_{1}}\right)_{s}=-\frac{1}{R^{\prime}}
\end{aligned}
$$

In (39)

$$
\begin{aligned}
& \left(\frac{\partial K}{\partial h_{0}}\right)_{s} \\
& \quad=-\frac{b_{0} A n}{H}\left[\frac{b_{0}\left(H-\bar{h}_{0}\right)}{H}+\frac{b_{1}\left(H-\bar{h}_{1}\right)}{H}\right]^{n-1} ; \\
& \left(\frac{\partial K}{\partial h_{1}}\right)_{s} \\
& =-\frac{b_{1} A n}{H}\left[\frac{b_{0}\left(H-\bar{h}_{0}\right)}{H}+\frac{b_{1}\left(H-\bar{h}_{1}\right)}{H}\right]^{n-1} .
\end{aligned}
$$

In (41)

$$
\begin{aligned}
\left(\frac{\partial Q_{p}}{\partial h_{0}}\right)_{s}= & \frac{1.79 \mu_{m} \sqrt{R^{\prime}}\left(-(1 / 2) \bar{h}_{0}+(3 / 2) \bar{h}_{1}\right)}{\bar{h}_{0}^{5 / 2}} \\
& -\frac{1.02 \bar{h}_{1}}{\bar{h}_{0}^{2}} ; \\
\left(\frac{\partial Q_{p}}{\partial h_{1}}\right)_{s}= & \frac{-1.79 \mu_{m} \sqrt{R^{\prime}}}{\bar{h}_{0}^{3 / 2}}+\frac{1.02}{\bar{h}_{0}} .
\end{aligned}
$$

In (36)

$$
\begin{aligned}
\left(\frac{\partial h_{1}}{\partial \dot{y}_{c}}\right)_{s} & =\left(\frac{\partial h_{1}}{\partial v_{r}}\right)_{s}=\left(\frac{\partial h_{1}}{\partial x_{n}}\right)_{s}=\left(\frac{\partial h_{1}}{\partial x_{c}}\right)_{s} \\
& =\left(\frac{\delta h_{1}}{\delta \dot{x}_{c}}\right)_{s}=0 ; \\
\left(\frac{\partial h_{1}}{\partial h_{c}}\right)_{s} & =1 .
\end{aligned}
$$

$$
\begin{aligned}
& a_{x_{n}, \sigma_{0}}=\left(\frac{\partial x_{n}}{\partial \sigma_{0}}\right)_{s}+0.7\left(\frac{\partial x_{n}}{\partial \sigma_{m}}\right)_{s} ; \\
& a_{x_{n}, \sigma_{1}}=\left(\frac{\partial x_{n}}{\partial \sigma_{1}}\right)_{s}+0.3\left(\frac{\partial x_{n}}{\partial \sigma_{m}}\right)_{s} ;
\end{aligned}
$$




$$
\begin{aligned}
a_{x_{n}, y_{c}}= & \left(\frac{\partial x_{n}}{\partial \delta}\right)_{s}\left(\frac{\partial \delta}{\partial x_{1}}\right)_{s}\left(\frac{\partial x_{1}}{\partial \dot{y}_{c}}\right)_{s} ; \\
a_{x_{n}, h_{0}}= & \left(\frac{\partial x_{n}}{\partial h_{0}}\right)_{s}+\left(\frac{\partial x_{n}}{\partial \alpha}\right)_{s}\left(\frac{\partial \alpha}{\partial h_{0}}\right)_{s} \\
& +\left(\frac{\partial x_{n}}{\partial K}\right)_{s}\left(\frac{\partial K}{\partial h_{0}}\right)_{s} \\
& +\left(\frac{\partial x_{n}}{\partial Q_{p}}\right)_{s}\left(\frac{\partial Q_{p}}{\partial h_{0}}\right)_{s} \\
a_{x_{n}, y_{c}}= & 2\left(\frac{\partial x_{n}}{\partial h_{1}}\right)_{s}\left(\frac{\partial h_{1}}{\partial h_{c}}\right)_{s} \\
& +2\left(\frac{\partial x_{n}}{\partial K}\right)_{s}\left(\frac{\partial K}{\partial h_{1}}\right)_{s}\left(\frac{\partial h_{1}}{\partial h_{c}}\right)_{s} \\
& +2\left(\frac{\partial x_{n}}{\partial \alpha}\right)_{s}\left(\frac{\partial \alpha}{\partial h_{c}}\right)_{s} \\
& +\left(\frac{\partial x_{n}}{\partial \delta}\right)_{s}\left(\frac{\partial \delta}{\partial x_{1}}\right)_{s}\left(\frac{\partial x_{1}}{\partial x_{c}}\right)_{s} \\
& +2\left(\frac{\partial x_{n}}{\partial Q_{p}}\right)_{s}\left(\frac{\partial Q_{p}}{\partial h_{1}}\right)_{s}\left(\frac{\partial h_{1}}{\partial h_{c}}\right)_{s} ; \\
& \left(\frac{\partial x_{n}}{\partial x_{c}}\right)_{s}\left(\frac{\partial x_{n}}{\partial \delta}\right)_{s}\left(\frac{\partial \delta}{\partial x_{c}}\right)_{s}
\end{aligned}
$$

In (44)

$$
\begin{aligned}
& \left(\frac{\partial v_{1}}{\partial h_{0}}\right)_{s}=\frac{\bar{v}_{0}}{\bar{h}_{c}} \\
& \left(\frac{\partial v_{1}}{\partial h_{c}}\right)_{s}=-\frac{\bar{v}_{0} \bar{h}_{0}}{\bar{h}_{c}^{2}} ; \\
& \left(\frac{\partial v_{1}}{\partial \dot{y}_{c}}\right)_{s}=\frac{2 \bar{x}_{0}}{\bar{h}_{c}} \\
& \left(\frac{\partial v_{1}}{\partial x_{c}}\right)_{s}=\left(\frac{\partial v_{1}}{\partial x_{1}}\right)_{s}=\left(\frac{\partial v_{1}}{\partial x_{0}}\right)_{s}=0 \\
& \left(\frac{\partial v_{1}}{\partial v_{0}}\right)_{s}=\frac{\bar{h}_{0}}{\bar{h}_{c}} ; \\
& \left(\frac{\partial v_{1}}{\partial \dot{x}_{c}}\right)_{s}=-\frac{\bar{h}_{0}-\bar{h}_{c}}{\bar{h}_{c}} .
\end{aligned}
$$

In (45)

In (43)

$$
\begin{aligned}
& \left(\frac{\partial v_{0}}{\partial h_{0}}\right)_{s}=-\frac{\bar{v}_{r} \bar{h}_{n}}{\bar{h}_{0}^{2}} ; \\
& \left(\frac{\partial v_{0}}{\partial h_{c}}\right)_{s}=\frac{\bar{v}_{r}}{\bar{h}_{0}} ; \\
& \left(\frac{\partial v_{0}}{\partial \dot{y}_{c}}\right)_{s}=\frac{2\left(\bar{x}_{n}-\bar{x}_{0}\right)}{\bar{h}_{0}} ; \\
& \left(\frac{\partial v_{0}}{\partial x_{c}}\right)_{s}=-\frac{2 \bar{v}_{r} \bar{x}_{n}}{R^{\prime} \bar{h}_{0}} ; \\
& \left(\frac{\partial v_{0}}{\partial \dot{x}_{c}}\right)_{s}=\frac{\bar{h}_{0}-\bar{h}_{n}}{\bar{h}_{0}} ; \\
& \left(\frac{\partial v_{0}}{\partial x_{0}}\right)_{s}=0 ; \\
& \left(\frac{\partial v_{0}}{\partial v_{r}}\right)_{s}=\frac{\bar{h}_{n}}{\bar{h}_{0}} \\
& \left(\frac{\partial v_{0}}{\partial x_{n}}\right)_{s}=\frac{2 \bar{v}_{r} \bar{x}_{n}}{R^{\prime} \bar{h}_{0}}
\end{aligned}
$$

In (46)

$$
\begin{aligned}
& a_{v_{1}, \sigma_{0}}=a_{v_{0}, \sigma_{0}}\left(\frac{\partial v_{1}}{\partial v_{0}}\right)_{s} ; \\
& a_{v_{1}, \sigma_{1}}=a_{v_{0}, \sigma_{1}}\left(\frac{\partial v_{1}}{\partial v_{0}}\right)_{s} ; \\
& a_{v_{1}, h_{0}}=\left(\frac{\partial v_{1}}{\partial h_{0}}\right)_{s}+a_{v_{0}, h_{0}}\left(\frac{\partial v_{1}}{\partial v_{0}}\right)_{s} ; \\
& a_{v_{1}, y_{c}}=2\left(\frac{\partial v_{1}}{\partial h_{c}}\right)_{s}+a_{v_{0}, y_{c}}\left(\frac{\partial v_{1}}{\partial v_{0}}\right)_{s} ;
\end{aligned}
$$




$$
\begin{aligned}
& a_{v_{1}, \dot{y}_{c}}=\left(\frac{\partial v_{1}}{\partial \dot{y}_{c}}\right)_{s}+a_{v_{0}, \dot{y}_{c}}\left(\frac{\partial v_{1}}{\partial v_{0}}\right)_{s} ; \\
& a_{v_{1}, x_{c}}=a_{v_{0}, x_{c}}\left(\frac{\partial v_{1}}{\partial v_{0}}\right)_{s} ; \\
& a_{v_{1}, \dot{x}_{c}}=\left(\frac{\partial v_{1}}{\partial \dot{x}_{c}}\right)_{s}+a_{v_{0}, \dot{x}_{c}}\left(\frac{\partial v_{1}}{\partial v_{0}}\right)_{s} ; \\
& a_{v_{1}, \dot{\theta}_{M}}=a_{v_{0}, \dot{\theta}_{M}}\left(\frac{\partial v_{1}}{\partial v_{0}}\right)_{s} .
\end{aligned}
$$

In (47)

$$
\begin{aligned}
\left(\frac{\partial F_{x}}{\partial \sigma_{0}}\right)_{s} & =-\frac{\bar{h}_{0} B}{2} ; \\
\left(\frac{\partial F_{x}}{\partial \sigma_{1}}\right)_{s} & =\frac{\bar{h}_{1} B}{2}=\frac{\bar{h}_{c} B}{2} ; \\
\left(\frac{\partial F_{x}}{\partial h_{0}}\right)_{s} & =-\frac{\bar{\sigma}_{0} B}{2} ; \\
\left(\frac{\partial F_{x}}{\partial h_{1}}\right)_{s} & =\frac{\bar{\sigma}_{1} B}{2} ; \\
a_{F_{x}, \sigma_{0}} & =\left(\frac{\partial F_{x}}{\partial \sigma_{0}}\right)_{s} \\
a_{F_{x}, \sigma_{1}} & =\left(\frac{\partial F_{x}}{\partial \sigma_{1}}\right)_{s} \\
a_{F_{x}, h_{0}} & =\left(\frac{\partial F_{x}}{\partial h_{0}}\right)_{s} ; \\
a_{F_{x}, y_{c}} & =2\left(\frac{\partial F_{x}}{\partial h_{1}}\right)_{s}\left(\frac{\partial h_{1}}{\partial h_{c}}\right)_{s} .
\end{aligned}
$$

In (48)

$$
\begin{aligned}
\left(\frac{\partial F_{y}}{\partial K}\right)_{s} & =B \bar{Q}_{p} \bar{l} ; \\
\left(\frac{\partial F_{y}}{\partial \sigma_{m}}\right)_{s} & =-B \bar{Q}_{p} \bar{l} ; \\
\left(\frac{\partial F_{y}}{\partial Q_{p}}\right)_{s} & =\left(\bar{K}-\bar{\sigma}_{m}\right) B \bar{l} ; \\
\left(\frac{\partial F_{y}}{\partial l}\right)_{s} & =\left(\bar{K}-\bar{\sigma}_{m}\right) B \bar{Q}_{p} ; \\
a_{F_{y}, \sigma_{0}} & =0.7\left(\frac{\partial F_{y}}{\partial \sigma_{m}}\right)_{s} \\
a_{F_{y}, \sigma_{1}} & =0.3\left(\frac{\partial F_{y}}{\partial \sigma_{m}}\right)_{s}
\end{aligned}
$$

$$
\begin{aligned}
a_{F_{y}, h_{0}}= & \left(\frac{\partial F_{y}}{\partial K}\right)_{s}\left(\frac{\partial K}{\partial h_{0}}\right)_{s}+\left(\frac{\partial F_{y}}{\partial Q_{p}}\right)_{s}\left(\frac{\partial Q_{p}}{\partial h_{0}}\right)_{s} \\
& -\left(\frac{\partial F_{y}}{\partial l}\right)_{s}\left(\frac{\partial x_{0}}{\partial h_{0}}\right)_{s} ; \\
a_{F_{y}, y_{c}}= & 2\left(\frac{\partial F_{y}}{\partial K}\right)_{s}\left(\frac{\partial K}{\partial h_{1}}\right)_{s}\left(\frac{\partial h_{1}}{\partial h_{c}}\right)_{s} \\
& +2\left(\frac{\partial F_{y}}{\partial Q_{p}}\right)_{s}\left(\frac{\partial Q_{p}}{\partial h_{1}}\right)_{s}\left(\frac{\partial h_{1}}{\partial h_{c}}\right)_{s} \\
& -2\left(\frac{\partial F_{y}}{\partial l}\right)_{s}\left(\frac{\partial x_{0}}{\partial h_{c}}\right)_{s} ; \\
a_{F_{y}, y_{c}}= & \left(\frac{\partial F_{y}}{\partial l}\right)_{s}\left(\frac{\partial x_{1}}{\partial \dot{y}_{c}}\right)_{s} ; \\
a_{F_{y}, x_{c}}= & \left(\frac{\partial F_{y}}{\partial l}\right)_{s}\left(\frac{\partial x_{1}}{\partial x_{c}}\right)_{s}-\left(\frac{\partial F_{y}}{\partial l}\right)_{s}\left(\frac{\partial x_{0}}{\partial x_{c}}\right)_{s} .
\end{aligned}
$$

In (49)

$$
\begin{aligned}
& \left(\frac{\partial \gamma}{\partial x_{c}}\right)_{s}=\frac{1}{R^{\prime}} \\
& \left(\frac{\partial \gamma}{\partial x_{n}}\right)_{s}=-\frac{1}{R^{\prime}}
\end{aligned}
$$

In (50)

$$
\begin{aligned}
& \left(\frac{\partial M}{\partial \alpha}\right)_{s}=-\frac{2 \bar{\gamma}}{\bar{\alpha}^{2}} \mu_{m}\left(\bar{K}-\bar{\sigma}_{m}\right) B \bar{Q}_{p} \bar{l} R \\
& \left(\frac{\partial M}{\partial \gamma}\right)_{s}=\frac{2}{\bar{\alpha}} \mu_{m}\left(\bar{K}-\bar{\sigma}_{m}\right) B \overline{Q_{p}} \bar{l} R \\
& \left(\frac{\partial M}{\partial K}\right)_{s}=-\frac{\bar{\alpha}-2 \bar{\gamma}}{\bar{\alpha}} \mu_{m} B \overline{Q_{p}} \bar{l} R ; \\
& \left(\frac{\partial M}{\partial \delta}\right)_{s}=-\frac{2 \bar{\alpha}-2 \bar{\gamma}}{\bar{\alpha}} \mu_{m}\left(\bar{K}-\bar{\sigma}_{m}\right) B \bar{Q}_{p} \bar{l} R \\
& \left(\frac{\partial M}{\partial \sigma_{m}}\right)_{s}=\frac{\bar{\alpha}-2 \bar{\gamma}}{\bar{\alpha}} \mu_{m} B \bar{Q}_{p} \bar{l} R ; \\
& \left(\frac{\partial M}{\partial Q_{p}}\right)_{s}=-\frac{\bar{\alpha}-2 \bar{\gamma}}{\bar{\alpha}} \mu_{m}\left(\bar{K}-\bar{\sigma}_{m}\right) B \bar{l} R ; \\
& \left(\frac{\partial M}{\partial l}\right)_{s}=-\frac{\bar{\alpha}-2 \bar{\gamma}}{\bar{\alpha}} \mu_{m}\left(\bar{K}-\bar{\sigma}_{m}\right) B \bar{Q}_{p} R ; \\
& a_{M, \sigma_{0}}=a_{x_{n}, \sigma_{0}}\left(\frac{\partial M}{\partial \gamma}\right)_{s}\left(\frac{\partial \gamma}{\partial x_{n}}\right)_{s}+0.7\left(\frac{\partial M}{\partial \sigma_{m}}\right)_{s} \\
& a_{M, \sigma_{1}}=a_{x_{n}, \sigma_{1}}\left(\frac{\partial M}{\partial \gamma}\right)_{s}\left(\frac{\partial \gamma}{\partial x_{n}}\right)_{s}+0.3\left(\frac{\partial M}{\partial \sigma_{m}}\right)_{s}
\end{aligned}
$$


TABle 6

\begin{tabular}{|c|c|c|c|c|c|c|c|c|c|}
\hline Number & $h_{0}(\mathrm{~mm})$ & $h_{1}(\mathrm{~mm})$ & $T_{0}(\mathrm{kN})$ & $T_{1}(\mathrm{kN})$ & $B(\mathrm{~m})$ & $A(\mathrm{MPa})$ & $H(\mathrm{~mm})$ & $P(\mathrm{kN})$ & $M(\mathrm{kN} \cdot \mathrm{m})$ \\
\hline 1 & 0.571 & 0.423 & 80.4 & 63.8 & 1.005 & 775 & 2.25 & 7272 & 19.0 \\
\hline 2 & 0.590 & 0.444 & 100.3 & 78.1 & 1.173 & 747 & 2.25 & 7899 & 20.5 \\
\hline 3 & 0.621 & 0.468 & 87.3 & 70.5 & 1.004 & 730 & 2.25 & 6641 & 17.5 \\
\hline 4 & 0.668 & 0.505 & 118.8 & 93.0 & 1.271 & 724 & 2.25 & 8241 & 22.5 \\
\hline 5 & 0.668 & 0.505 & 118.8 & 93.0 & 1.271 & 723 & 2.25 & 8227 & 22.0 \\
\hline 6 & 0.590 & 0.444 & 100.3 & 78.1 & 1.173 & 754 & 2.25 & 8007 & 21.5 \\
\hline 7 & 0.589 & 0.443 & 100.3 & 78.0 & 1.173 & 761 & 2.25 & 8157 & 21.5 \\
\hline 8 & 0.590 & 0.444 & 100.4 & 78.1 & 1.173 & 737 & 2.25 & 7743 & 20.0 \\
\hline 9 & 0.590 & 0.444 & 100.4 & 78.1 & 1.173 & 760 & 2.25 & 7644 & 20.0 \\
\hline 10 & 0.590 & 0.444 & 100.3 & 78.1 & 1.173 & 760 & 2.25 & 7630 & 19.5 \\
\hline 11 & 0.571 & 0.423 & 80.4 & 63.8 & 1.005 & 774 & 2.25 & 7269 & 19.0 \\
\hline 12 & 0.620 & 0.467 & 87.2 & 70.4 & 1.004 & 752 & 2.25 & 6582 & 17.5 \\
\hline 13 & 0.588 & 0.442 & 100.0 & 77.8 & 1.173 & 741 & 2.25 & 7304 & 19.0 \\
\hline 14 & 0.621 & 0.468 & 87.2 & 70.4 & 1.004 & 730 & 2.25 & 6410 & 17.0 \\
\hline 15 & 0.621 & 0.468 & 87.2 & 70.4 & 1.004 & 734 & 2.25 & 6579 & 18.0 \\
\hline 16 & 0.620 & 0.467 & 87.2 & 70.4 & 1.004 & 750 & 2.25 & 6585 & 18.0 \\
\hline 17 & 0.588 & 0.442 & 100.0 & 77.8 & 1.173 & 749 & 2.25 & 7841 & 20.0 \\
\hline 18 & 0.621 & 0.468 & 87.2 & 70.4 & 1.004 & 727 & 2.25 & 6287 & 17.5 \\
\hline 19 & 0.621 & 0.465 & 87.2 & 70.4 & 1.004 & 731 & 2.25 & 6524 & 18.0 \\
\hline 20 & 0.620 & 0.467 & 87.2 & 70.4 & 1.004 & 743 & 2.25 & 6458 & 18.0 \\
\hline 21 & 0.588 & 0.442 & 100.0 & 77.8 & 1.173 & 752 & 2.25 & 7549 & 20.0 \\
\hline 22 & 0.571 & 0.423 & 80.4 & 63.8 & 1.005 & 771 & 2.25 & 6999 & 18.5 \\
\hline 23 & 0.588 & 0.442 & 100.0 & 77.8 & 1.173 & 744 & 2.25 & 7765 & 20.0 \\
\hline 24 & 0.620 & 0.467 & 87.2 & 70.4 & 1.004 & 748 & 2.25 & 6699 & 18.0 \\
\hline 25 & 0.588 & 0.442 & 100.1 & 77.8 & 1.173 & 721 & 2.25 & 7620 & 18.5 \\
\hline 26 & 0.571 & 0.423 & 80.4 & 63.8 & 1.005 & 773 & 2.25 & 7282 & 19.0 \\
\hline 27 & 0.571 & 0.423 & 80.4 & 63.8 & 1.005 & 753 & 2.25 & 6617 & 17.0 \\
\hline 28 & 0.571 & 0.423 & 80.3 & 63.8 & 1.005 & 772 & 2.25 & 6980 & 18.0 \\
\hline 29 & 0.571 & 0.423 & 80.3 & 63.8 & 1.005 & 766 & 2.25 & 6719 & 17.5 \\
\hline 30 & 0.621 & 0.468 & 87.2 & 70.4 & 1.004 & 733 & 2.25 & 6199 & 16.5 \\
\hline 31 & 0.621 & 0.468 & 87.2 & 70.4 & 1.004 & 732 & 2.25 & 6339 & 17.0 \\
\hline 32 & 0.588 & 0.442 & 100.0 & 77.8 & 1.173 & 748 & 2.25 & 7164 & 18.5 \\
\hline 33 & 0.621 & 0.468 & 87.2 & 70.4 & 1.004 & 729 & 2.25 & 6079 & 16.5 \\
\hline 34 & 0.647 & 0.496 & 103.2 & 81.8 & 1.100 & 725 & 2.25 & 6536 & 18.0 \\
\hline 35 & 0.588 & 0.442 & 100.0 & 77.8 & 1.173 & 742 & 2.25 & 7244 & 19.5 \\
\hline 36 & 0.571 & 0.423 & 80.4 & 63.8 & 1.005 & 764 & 2.25 & 6712 & 18.5 \\
\hline 37 & 0.571 & 0.423 & 80.3 & 63.8 & 1.005 & 766 & 2.25 & 7181 & 20.0 \\
\hline 38 & 0.571 & 0.423 & 80.4 & 63.8 & 1.005 & 770 & 2.25 & 7207 & 19.5 \\
\hline 39 & 0.621 & 0.468 & 87.3 & 70.5 & 1.004 & 711 & 2.25 & 6257 & 17.0 \\
\hline 40 & 0.571 & 0.423 & 80.4 & 63.8 & 1.005 & 748 & 2.25 & 6811 & 18.0 \\
\hline 41 & 0.648 & 0.496 & 100.3 & 81.9 & 1.100 & 718 & 2.25 & 6739 & 18.5 \\
\hline 42 & 0.621 & 0.468 & 87.2 & 70.4 & 1.004 & 726 & 2.25 & 6419 & 18.0 \\
\hline 43 & 0.621 & 0.468 & 87.2 & 70.4 & 1.004 & 721 & 2.25 & 6105 & 16.5 \\
\hline 44 & 0.621 & 0.465 & 87.2 & 70.4 & 1.004 & 715 & 2.25 & 6038 & 16.5 \\
\hline 45 & 0.571 & 0.423 & 80.3 & 63.8 & 1.005 & 755 & 2.25 & 6751 & 18.0 \\
\hline 46 & 0.571 & 0.423 & 80.3 & 63.8 & 1.005 & 763 & 2.25 & 7119 & 19.0 \\
\hline 47 & 0.571 & 0.423 & 80.4 & 63.8 & 1.005 & 743 & 2.25 & 6924 & 19.0 \\
\hline 48 & 0.570 & 0.422 & 80.2 & 63.7 & 1.005 & 728 & 2.25 & 6339 & 17.0 \\
\hline 49 & 0.647 & 0.496 & 103.2 & 81.8 & 1.100 & 721 & 2.25 & 6712 & 19.0 \\
\hline 50 & 0.580 & 0.422 & 83.7 & 65.2 & 1.030 & 730 & 2.25 & 6907 & 20.0 \\
\hline
\end{tabular}




$$
\begin{aligned}
& a_{M, h_{0}}=\left(\frac{\partial M}{\partial \alpha}\right)_{s}\left(\frac{\partial \alpha}{\partial h_{0}}\right)_{s} \\
& +a_{x_{n}, h_{0}}\left(\frac{\partial M}{\partial \gamma}\right)_{s}\left(\frac{\partial \gamma}{\partial x_{n}}\right)_{s} \\
& +\left(\frac{\partial M}{\partial K}\right)_{s}\left(\frac{\partial K}{\partial h_{0}}\right)_{s} \\
& +\left(\frac{\partial M}{\partial Q_{p}}\right)_{s}\left(\frac{\partial Q_{p}}{\partial h_{0}}\right)_{s} \\
& -\left(\frac{\partial M}{\partial l}\right)_{s}\left(\frac{\partial x_{0}}{\partial h_{0}}\right)_{s} ; \\
& a_{M, y_{c}}=2\left(\frac{\partial M}{\partial \alpha}\right)_{s}\left(\frac{\partial \alpha}{\partial h_{c}}\right)_{s} \\
& +a_{x_{n}, y_{c}}\left(\frac{\partial M}{\partial \gamma}\right)_{s}\left(\frac{\partial \gamma}{\partial x_{n}}\right)_{s} \\
& +2\left(\frac{\partial M}{\partial K}\right)_{s}\left(\frac{\partial K}{\partial h_{1}}\right)_{s}\left(\frac{\partial h_{1}}{\partial h_{c}}\right)_{s} \\
& +2\left(\frac{\partial M}{\partial Q_{p}}\right)_{s}\left(\frac{\partial Q_{p}}{\partial h_{1}}\right)_{s}\left(\frac{\partial h_{1}}{\partial h_{c}}\right)_{s} \\
& -2\left(\frac{\partial M}{\partial l}\right)_{s}\left(\frac{\partial x_{0}}{\partial h_{c}}\right)_{s} ; \\
& a_{M, \dot{y}_{c}}=a_{x_{n}, \dot{y}_{c}}\left(\frac{\partial M}{\partial \gamma}\right)_{s}\left(\frac{\partial \gamma}{\partial x_{n}}\right)_{s} \\
& +\left(\frac{\partial M}{\partial \delta}\right)_{s}\left(\frac{\partial \delta}{\partial x_{1}}\right)_{s}\left(\frac{\partial x_{1}}{\partial \dot{y}_{c}}\right)_{s} \\
& +\left(\frac{\partial M}{\partial l}\right)_{s}\left(\frac{\partial x_{1}}{\partial \dot{y}_{c}}\right)_{s} ; \\
& a_{M, x_{c}}=\left(\frac{\partial M}{\partial \gamma}\right)_{s}\left(\frac{\partial \gamma}{\partial x_{c}}\right)_{s} \\
& +a_{x_{n}, x_{c}}\left(\frac{\partial M}{\partial \gamma}\right)_{s}\left(\frac{\partial \gamma}{\partial x_{n}}\right)_{s} \\
& +\left(\frac{\partial M}{\partial \delta}\right)_{s}\left(\frac{\partial \delta}{\partial x_{c}}\right)_{s} \\
& +\left(\frac{\partial M}{\partial \delta}\right)_{s}\left(\frac{\partial \delta}{\partial x_{1}}\right)_{s}\left(\frac{\partial x_{1}}{\partial x_{c}}\right)_{s} \\
& +\left(\frac{\partial M}{\partial l}\right)_{s}\left(\frac{\partial x_{1}}{\partial x_{c}}\right)_{s} \\
& -\left(\frac{\partial M}{\partial l}\right)_{s}\left(\frac{\partial x_{0}}{\partial x_{c}}\right)_{s} \text {. }
\end{aligned}
$$

\section{B. Experimental Data from the 4th Stand of a Five-Stand Cold Tandem Mill}

See Table 6.

\section{Competing Interests}

The authors declare that they have no competing interests.

\section{Acknowledgments}

The supports of the National Natural Science Foundation of China (no. 51175035), the Ph.D. Programs Foundation of Ministry of Education of China (no. 20100006110024), the Beijing Higher Education Young Elite Teacher Project (no. YETP0367), and the Fundamental Research Funds for the Central Universities (no. FRF-BR-14-006A) are gratefully acknowledged.

\section{References}

[1] I. Yarita, K. Furukawa, and Y. Seino, "Analysis of chattering in cold rolling for ultra-thin gauge steel strip," Transctions ISIJ, vol. 18, no. 1, pp. 1-10, 1978.

[2] T. Tamiya, K. Furui, and H. Lida, "Analysis of chattering phenomenon in cold rolling," in Proceedings of the International Conference on Steel Rolling, vol. 2, pp. 1191-1202, Tokyo, Japan, 1980.

[3] I. S. Yun, W. R. D. Wilson, and K. F. Ehmann, "Chatter in the strip rolling process, part 1: dynamic model of rolling; part 2: dynamic rolling experiments; part 3: chatter model," Journal of Manufacturing Science and Engineering, vol. 120, no. 5, pp. 330348, 1998.

[4] P. H. Hu, Y. H. Zhao, and K. F. Ehmann, “Third-octave-mode chatter in rolling, part 1: chatter model; part 2: stability of a single-stand mill; part 3: stability of a multi-stand mill," Proceedings of the Institution of Mechanical Engineers, Part B: Journal of Engineering Manufacture, vol. 220, no. 8, pp. 12671303, 2006.

[5] H. Zhao and K. F. Ehmann, "Stability analysis of chatter in tandem rolling mills-part 1: single- and multi-stand negative damping effect," Journal of Manufacturing Science and Engineering, vol. 135, no. 3, Article ID 031001, pp. 1-19, 2013.

[6] P. A. Meehan, "Vibration instability in rolling mills: modeling and experimental results," Journal of Vibration and Acoustics, vol. 124, no. 2, pp. 221-228, 2002.

[7] W. L. Roberts, "Four-h mill-stand chatter of the fifth-octave mode," Iron and Steel Engineer, vol. 55, no. 10, pp. 41-47, 1978.

[8] P.-H. Hu and K. F. Ehmann, "Fifth octave mode chatter in rolling," Proceedings of the Institution of Mechanical Engineers, Part B: Journal of Engineering Manufacture, vol. 215, no. 6, pp. 797-809, 2001.

[9] J. H. Gallenstein, “Torsional chatter on a 4-h cold mill,” Iron and Steel Engineer, vol. 58, no. 1, pp. 52-57, 1981.

[10] P. Krot, "Nonlinear vibrations and backlashes diagnostics in the rolling mills drive trains," in Proceedings of the 6th EUROMECH Nonlinear Dynamics Conference (ENOC '08), vol. 30, pp. 26-30, Saint Petersburg, Russia, 2008.

[11] D. L. Paton and S. Critchley, "Tandem mill vibration: its cause and control," Iron Steelmaker, vol. 12, no. 3, pp. 37-43, 1985. 
[12] G. X. Shen and M. Li, "Statically determinate characteristics of microdisplacement in a four-high mill," Journal of Materials Processing Technology, vol. 209, no. 11, pp. 5002-5007, 2009.

[13] S. K. Dwivedy, S. S. Dhutekar, and P. Eberhard, "Numerical investigation of chatter in cold rolling mills," in Materials with Complex Behaviour II, A. Öchsner, L. F. M. da Silva, and H. Altenbach, Eds., vol. 16 of Advanced Structured Materials, pp. 213-227, Springer, Berlin, Germany, 2012.

[14] A. Swiatoniowski, "Interdependence between rolling mill vibrations and the plastic deformation process," Journal of Materials Processing Technology, vol. 61, no. 4, pp. 354-364, 1996.

[15] X. Q. Yan, C. Shi, X. Cao, and L. Liu, "Research on coupled vertical-torsion vibration of mill-stand of CSP mill," Journal of Vibration, Measurement and Diagnosis, vol. 28, no. 4, pp. 377381, 2008.

[16] D. X. Hou, R. R. Peng, and H. R. Liu, "Analysis of verticalhorizontal coupling vibration characteristics of rolling mill rolls based on strip dynamic deformation process," Shock and Vibration, vol. 2014, Article ID 543793, 11 pages, 2014.

[17] I. S. Yun, W. R. D. Wilson, and K. F. Ehmann, "Review of chatter studies in cold rolling," International Journal of Machine Tools and Manufacture, vol. 38, no. 12, pp. 1499-1530, 1998.

[18] J. Tlusty, G. Chandra, S. Critchley, and D. Paton, "Chatter in cold rolling," CIRP Annals-Manufacturing Technology, vol. 31, no. 1, pp. 195-199, 1982.

[19] L. Q. Zeng, Y. Zang, Z. Y. Gao, and X. C. Liu, "Study on overall coupled modeling of the rolling mill," Journal of Mechanical Engineering, vol. 51, no. 14, pp. 46-53, 2015.

[20] S. Z. Wen and P. Huang, Principles of Tribology, Tsinghua University Press, Beijing, China, 2012.

[21] J. X. Zou and L. J. Xu, Tandem Mill Vibration Control, Metallurgical Industry Press, Beijing, China, 1998.

[22] J. X. Zou, Steel Rolling Machinery, Metallurgical Industry Press, Beijing, China, 1988. 


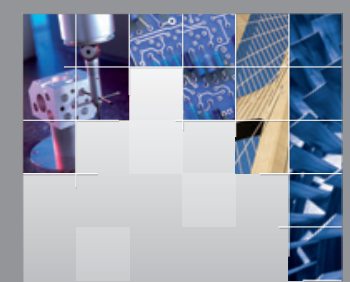

\section{Enfincering}
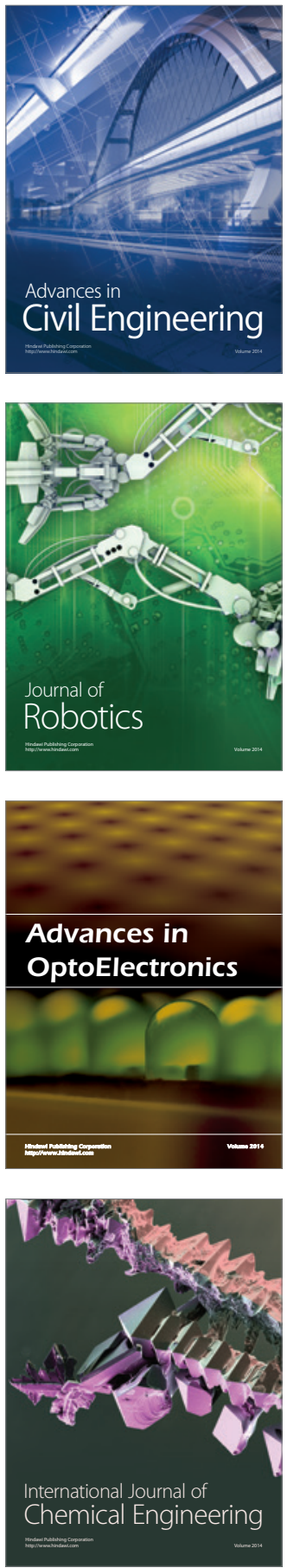

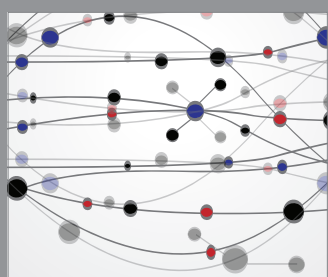

The Scientific World Journal

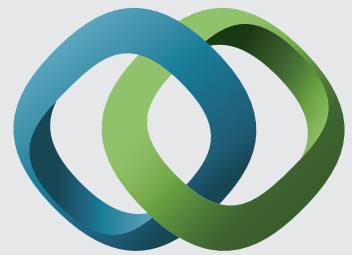

\section{Hindawi}

Submit your manuscripts at

http://www.hindawi.com
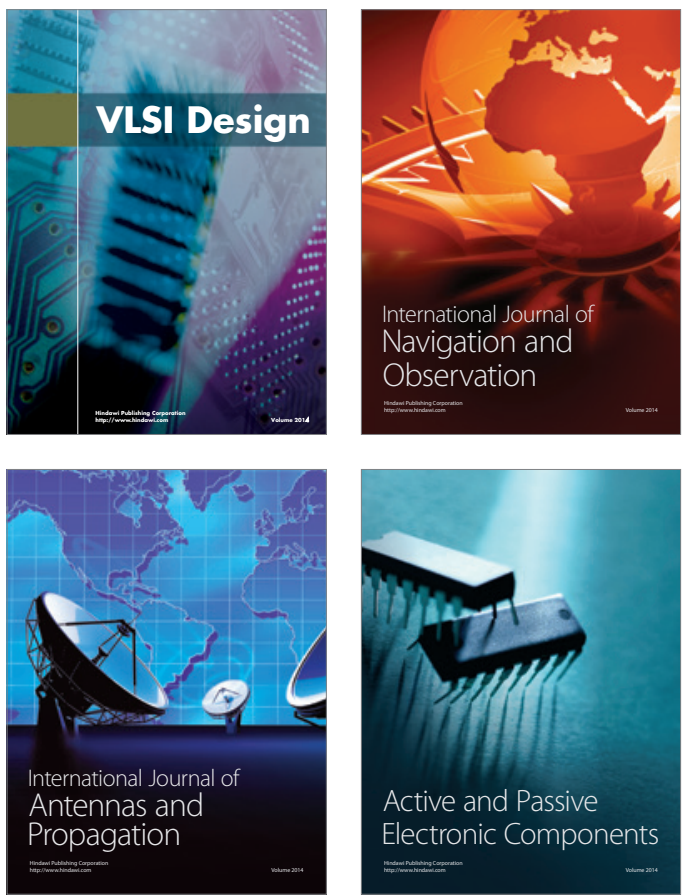
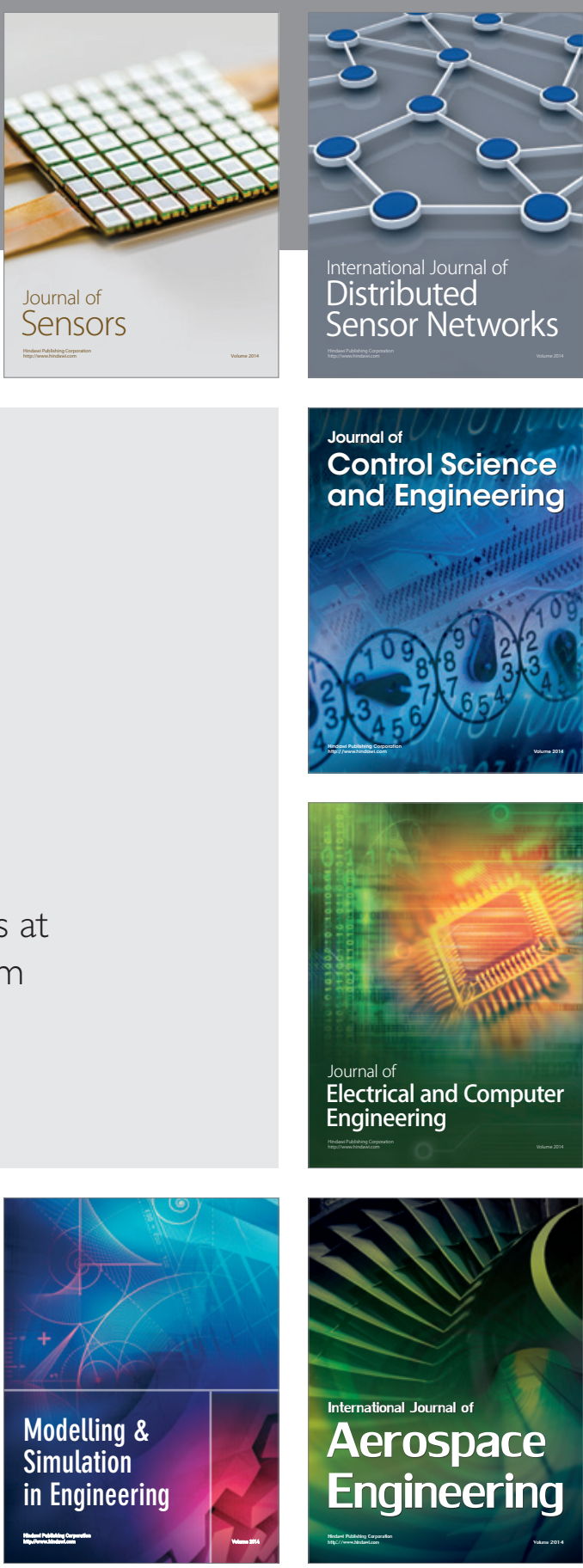

International Journal of

Distributed

Sensor Networks

Journal of

Control Science

and Engineering
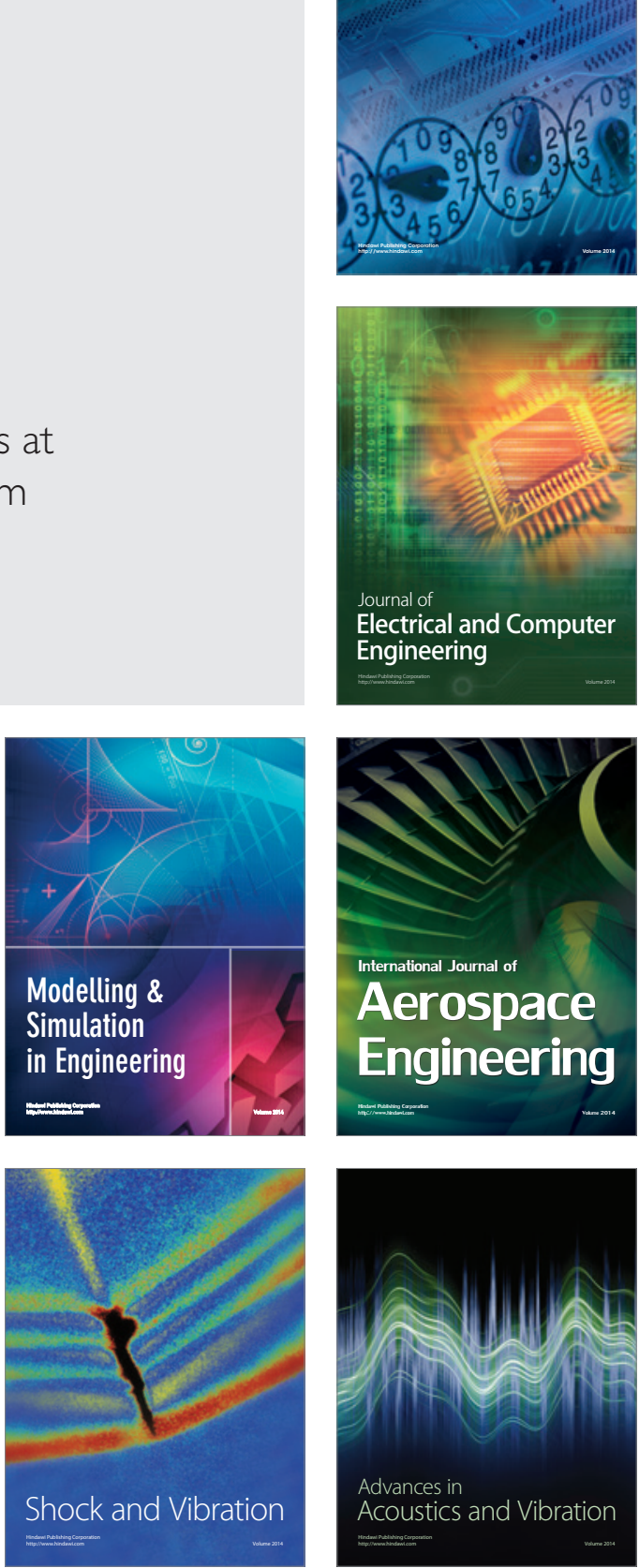